\title{
THE FREDERIKS EFFECT AND RELATED PHENOMENA IN FERRONEMATIC MATERIALS*
}

\author{
V. I. ZADOROZHNII ${ }^{\dagger}$, T. J. SLUCKIN ${ }^{\ddagger}$, V. YU. RESHETNYAK ${ }^{\dagger}$, AND K. S. THOMAS ${ }^{\S}$
}

\begin{abstract}
Using continuum and statistical mechanical theories, we study the switching properties of a ferronematic in a nematic liquid crystal cell subject to homeotropic boundary conditions at the cell and particle walls. An external magnetic field normal to the cell plane is also imposed. At low fields we find thresholdless switching of the nematic director, consistent with experimental data. At higher fields, there are three regimes, depending on the strength of the anchoring interaction between the director and the ferroparticle orientation. For low anchoring strengths, there is an inverse Frederiks effect, and the nematic reorientation reduces and then disappears continuously at a critical magnetic field. At intermediate fields, the degree of reorientation reduces at high fields but remains finite. For high fields, however, the director switching saturates. The dimensionless temperature scale in the problem involves the temperature, the mean nematic elastic constant, the colloidal density, and the cell dimension. If this quantity is sufficiently low, then high magnetic fields can cause magnetic segregation. The segregation order parameter is coupled to the director distortion, and this can change the inverse Frederiks transition into a first order transition, leading to bistability in an intermediate field regime. These features are perturbed but not changed structurally by the effect of a small bias magnetic field $(<10$ Oe) normal to the unperturbed director. Subject to suitable choice of parameters, the theory is also quantitatively consistent with the results of the classic experiment of Chen and Amer in 1983.
\end{abstract}

Key words. liquid crystals, ferronematics, colloids

AMS subject classifications. 82B26, 82D30, 82D45

DOI. $10.1137 / 070703831$

1. Introduction. In 1970 Brochard and de Gennes [1] suggested on theoretical grounds that it might be possible to construct magnetic colloids based on a liquid crystal matrix. As a result of the anchoring at the surface of the colloidal particles, magnetic and nematic order in these materials would be coupled. The weak interaction between magnetic fields and nematic order, already exploited by the early liquid crystal pioneers such as Charles Mauguin [2], would then be dramatically increased.

The giant magnetic-nematic coupling might then be fruitfully used in devices controlled by easily accessible magnetic fields $(<10 \mathrm{Oe})$. These systems, including as they do elements of both ferromagnetism and nematic liquid crystalline behavior, have come to be known as ferronematics (FNs). The key relevant properties of a nematic crystal doped with single-domain ferromagnetic particles are (a) the intrinsic high magnetic susceptibility and (b) the uniform molecular reorientation of the entire liquid crystal (LC) matrix, or macroscopic collective behavior [1], in a varying magnetic field.

It was not until 1983 that Chen and Amer [3] were first able to construct a model experimental system. One feature complicating the interpretation of experiments is

*Received by the editors September 26, 2007; accepted for publication (in revised form) March 28, 2008; published electronically July 2, 2008. INTAS (grant 99-00312) and NATO (grant CBP.NUKR.CLG.981968) have supported visits to Southampton by VYR and VIZ and visits to Kiev by TJS. We thank both these agencies for their continuing support.

http://www.siam.org/journals/siap/68-6/70383.html

${ }^{\dagger}$ Physics Faculty, Kyiv National Taras Shevchenko University, Prosp. Glushkova, 2, bldg 1, Kyiv, 03680, Ukraine (viza@mail.univ.kiev.ua, reshet@iop.kiev.ua).

${ }^{\ddagger}$ School of Mathematics, University of Southampton, Southampton, SO17 1BJ, United Kingdom (t.j.sluckin@soton.ac.uk).

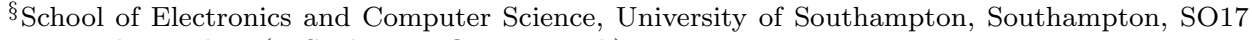
1BJ, United Kingdom (K.S.Thomas1@soton.ac.uk).

1688 
the fact that the ferroparticles can coagulate irreversibly. Indeed, subsequent work has continually been hindered for a long time by difficulties in the manufacture of stable reproducible magnetic liquid crystalline colloids.

In the last two decades interest in these materials has grown. Not only do these systems pose interesting physical problems, but also they promise to provide an optical device technology based on magnetic switching $[4,5,6,7,8]$. In particular, Buluy et al. [9] have recently synthesized an $\mathrm{FN}$ which is stable against colloidal aggregation. This system consists of magnetite particles $\left(\mathrm{Fe}_{3} \mathrm{O}_{4}\right)$ coated with oxyethyl-propylene glycol and suspended in 5CB.

The initial continuum theory [1] has been generalized by Burylov and Raikher $[10,11]$ to the case of a finite anchoring energy of the nematic at the ferroparticle surface. This provided an explanation for the absence of coalignment of the nematic director $\hat{\mathbf{n}}$ and the averaged local magnetization $\mathbf{M}$ in the $\mathrm{FN}$, as had been found experimentally [12]. Experiments, among them one on 8CB-based FN with magnetite particles of nearly spherical shape [6], have subsequently confirmed the generalized theory. The loss of coalignment has been labeled detachment in the literature. In this paper we determine some more precise conditions for detachment.

One other important feature which the theory must include is the possibility of magnetic segregation $[1,13]$. This involves the magnetic colloidal particles migrating toward regions in which the ferronematic coupling energy is minimized. Typically this occurs in the center of the sample, where the nematic director is most free to rotate toward the magnetically favored direction.

However, this tendency to segregate is opposed by entropic forces favoring a uniform colloidal concentration. The balance is subtle and is controlled by the dimensionless temperature, which is typically of order unity. Previous calculations [14] have suggested that this effect can lead to hysteretic effects as a function of magnetic field. The hysteretic effects have been interpreted as the signature of a decoupling of the nematic director and the ferroparticle orientation. In this paper we give a full account of the physics of the ordering process which occurs in a confined ferronematic system within the usual Frederiks geometry.

The plan of the paper is as follows. In section 2 we describe the theoretical model. In section 3 we give an analysis of the basic aspects of the model, specifically confining our interest to the case in which magnetic segregation is absent. Most of this work is analytical, but we conclude this section by presenting the results of some computational solutions of the equations. We also compare the numerical solutions and the analytical approximations; the analysis, even where only approximate, yields surprisingly accurate precisions. In section 4 we extend the model to include the so-called bias field. This is the extra in-plane magnetic field required to stabilize experimentally the systems we are discussing. In section 5 we add a discussion of magnetic segregation. Both bias field and magnetic segregation are important experimentally but might be regarded as complications to the basic underlying mathematical model. In section 6 we give a brief discussion linking our work to some of the available experiments. Finally, in section 7 we draw some conclusions and place our work in a larger context. A brief preliminary report of our work has been published elsewhere [15].

2. Model. The basic geometry of the problem is shown in Figure 1. The ferroparticles are needle-like monodomain ferrite grains of length $L$ and diameter $d \sim L / 7$ $L / 3$; they are significantly larger than the nematic molecule size. We consider a cell of thickness $D$ and suppose strong homeotropic nematic anchoring at the cell walls at $z=0$ and $z=D$. 


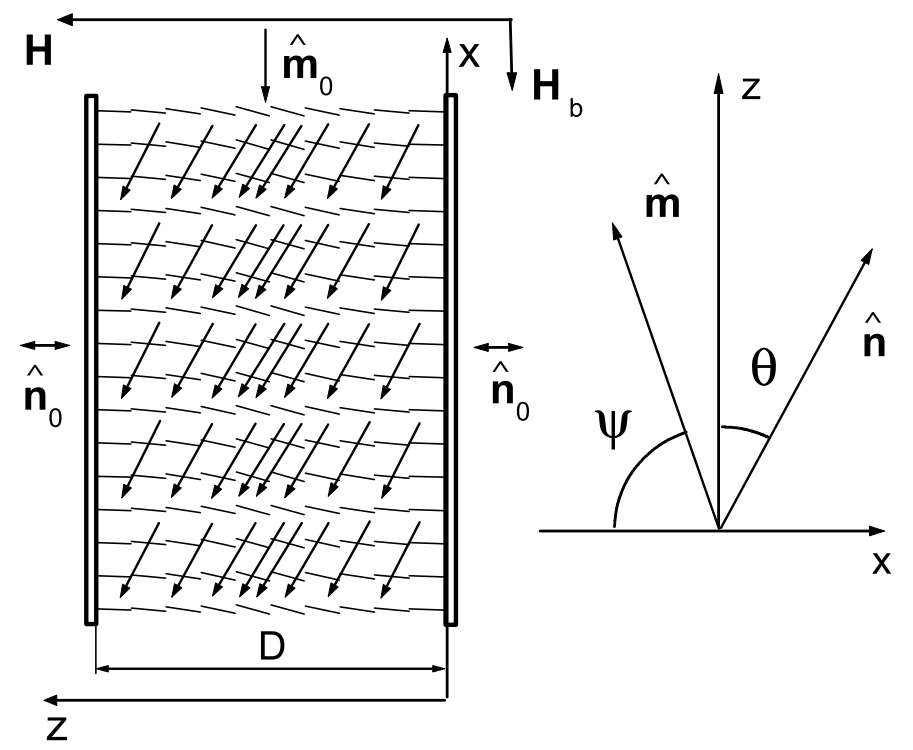

FIG. 1. Schematic of the FN cell. The total magnetic field is given by $\mathbf{H}_{s}=\left[H_{b}, 0, H\right]$, with $H_{b}$ the bias field and $H$ the imposed field. In the left-hand diagram, the magnetic director is marked with an arrow, while the nematic director is marked by a line. The meaning of the angles $\psi$, signifying the deviation of the magnetic director from its original orientation, and $\theta$, signifying the nematic deviation, are shown in the right-hand diagram.

Burylov and Raikher $[10,11]$ have derived an effective soft homeotropic anchoring energy $W_{p}$ per colloidal particle due to the nematic-ferroparticle surface interaction. We do not discuss the microscopic derivation of the magnitude of this interaction here but simply note that in our discussion it is a measurable parameter which enters the theory. The existence of this anchoring encourages, but does not oblige, the ferroparticles to orient perpendicular to the (averaged) nematic director. This term changes the physics of the classical nematic liquid crystal.

In addition, the cell may be initially subject to a small bias magnetic field $\mathbf{H}_{b}$ parallel to the cell substrates. In this initial state ferroparticles are homogeneously distributed over the cell volume and their magnetic moments are perpendicular to the unperturbed director $\hat{\mathbf{n}}_{0} . \hat{\mathbf{m}}_{0}$ is the unit vector in the direction of the sample magnetization at $\mathbf{H}=0$. The sample is uniform in the $x-y$ direction.

The physical states are defined by a nematic director $\hat{\mathbf{n}}(\mathbf{r})$, a local normalized magnetization $\hat{\mathbf{m}}(\mathbf{r})$, and a local colloidal particle density $\rho(\mathbf{r})=\frac{f(\mathbf{r})}{\nu}$, with $f$ the local colloidal packing fraction and $\nu$ the colloidal particle volume.

The FN equilibrium state at given magnetic field $\mathbf{H}_{s}=\mathbf{H}+\mathbf{H}_{b}$ can be determined by minimizing the full free energy functional [10]

$$
\begin{aligned}
\mathcal{F}= & \int_{V}\left\{\frac { 1 } { 2 } \left[K_{1}(\nabla \cdot \hat{\mathbf{n}})^{2}+K_{2}(\hat{\mathbf{n}} \cdot \nabla \times \hat{\mathbf{n}})^{2}\right.\right. \\
& \left.+K_{3}(\hat{\mathbf{n}} \times \nabla \times \hat{\mathbf{n}})^{2}\right]-\frac{1}{2} \chi_{a}\left(\hat{\mathbf{n}} \cdot \mathbf{H}_{s}\right)^{2}+\frac{f k_{B} T}{\nu} \ln f \\
& \left.-\bar{M} f\left(\hat{\mathbf{m}} \cdot \mathbf{H}_{s}\right)+f W_{p}(\hat{\mathbf{n}} \cdot \hat{\mathbf{m}})^{2}\right\} d V
\end{aligned}
$$

Copyright $@$ by SIAM. Unauthorized reproduction of this article is prohibited. 
subject to ferroparticle number conservation

$$
\int f d V=\bar{f} V
$$

where $K_{1}, K_{2}, K_{3}$ are elastic (Frank) constants, $\chi_{a}$ is the anisotropic part of the nematic diamagnetic susceptibility, $f$ and $\bar{f}$ are the local and the mean volume particle fractions, and $\hat{\mathbf{m}}$ is the unit vector in the direction of the sample magnetization $\mathbf{M}=\bar{M} f \hat{\mathbf{m}}$, where normally $\bar{M}=M_{s}$, the saturation magnetization per unit volume within an individual colloidal particle. In the limit of low applied fields, we shall find, however, that sometimes $\bar{M}$ is reduced from this saturation value by thermal fluctuation effects.

The term in square brackets in (1a) is the Frank-Oseen-Zocher curvature energy. The remaining terms are, respectively, the magnetic energy density, the contribution of the mixing entropy of an ideal ferroparticle solution, the magnetic energy of the colloidal particles, and the anchoring-induced ferronematic interaction [10]. We ignore the ferroparticle magnetic dipole-dipole interaction energy, which disappears at low ferroparticle concentrations. It will be useful to define the quantity $\eta(\mathbf{r})=\frac{f(\mathbf{r})}{\bar{f}}$. This is the local enhancement (or reduction) of the colloidal density induced by segregation effects. We also suppose the magnetization of the ferroparticles to have reached saturation [10].

In this geometry, the nematic and magnetic distortions are given, respectively, by $\hat{\mathbf{n}}=(\sin \theta, 0, \cos \theta)$ and $\hat{\mathbf{m}}=(-\cos \psi, 0, \sin \psi)$, where $\theta=\theta(z)$ and $\psi=\psi(z)$ are shown in Figure 1. In the simple case when the elastic constants $K_{1}$ and $K_{3}$ are taken to be equal $\left(K_{1}=K_{3}=K\right)$, the free energy functional reduces to

$$
\begin{aligned}
\mathcal{F}= & \int_{0}^{D}\left[\frac{1}{2} K\left(\frac{d \theta}{d z}\right)^{2}-\frac{1}{2} \chi_{a}\left(H \cos \theta-H_{b} \sin \theta\right)^{2}\right. \\
& +\eta(z) \frac{\bar{f} k_{B} T}{\nu} \ln \eta-\bar{M} \eta(z) \bar{f}\left(H \sin \psi+H_{b} \cos \psi\right) \\
& \left.+\eta(z) \bar{f} W_{p} \sin ^{2}(\theta-\psi)\right] d z,
\end{aligned}
$$

subject to the boundary condition $\theta(0)=\theta(D)=0$ and the constraint $\frac{1}{D} \int_{0}^{D} \eta(z) d z=$ 1.

We may sensibly enquire what conditions are required for (1a) and (2) to hold. This point has been addressed briefly by Burylov and Raikher [10, 11]. However, a detailed discussion of this point goes beyond the scope of this paper. The key point is that it should be possible to define the local magnetic director $\hat{\mathbf{m}}$ as a good macroscopic variable in a continuum theory. This in turn requires that there be effective coupling of the local magnetic directors over length scales larger than the typical interparticle distance. We return to this point briefly in section 6 , when we discuss the application of our work to experimental interpretation.

The effective coupling between the magnetic and nematic director will necessarily involve some orientational distortions induced by the colloidal particles over a length scale which may be considerably larger than the individual particle. A sufficient condition for the existence of the director $\hat{\mathbf{m}}$ will be that this distortion region be larger than the typical interparticle distance, so that the distortions induced by neighboring colloidal particles overlap. There will then be an indirect interaction between the 
magnetic particles, and a locally macroscopic structure. This condition has been termed the requirement for collective behavior $[1,10,16]$.

To simplify we initially suppose the following:

(a) There is no bias field. The raison d'être of the bias field is to hold $\hat{\mathbf{m}}$ and hence $\hat{\mathbf{n}}$ in the $x-z$ plane. In the absence of the bias field at very low applied fields $\bar{M} / M_{s}<1$, but here we shall suppose that this is not the case. We shall discuss the low bias field case (and criteria for determining what is meant by this limit) at the end of the paper.

(b) The direct magnetic-nematic interaction as a result of the anisotropic nematic molecular susceptibility can be ignored as compared to the indirect (but giant) colloidally mediated coupling. Normally, the anisotropic part of the nematic diamagnetic susceptibility is extremely low, for $5 \mathrm{CB} \chi_{a}=1.7 \times 10^{-7}[17]$. Thus neglecting the bare magnetic-nematic interaction is a reasonable approximation for the magnetic fields which we shall consider $(H<200 \mathrm{Oe})$, as we will see below.

We now nondimensionalize the problem. Length scales are now measured in units of the cell width $D$ (i.e., $z$ in scaled units is equal to $z / D$ in unscaled units). The scaled free energy is now given by the following formula:

$$
\begin{aligned}
F= & \int_{0}^{1} d z\left[\frac{1}{2}\left(\frac{d \theta}{d z}\right)^{2}+\eta t \ln \eta-\eta h \sin \psi\right. \\
& \left.+\eta w \sin ^{2}(\theta-\psi)\right]
\end{aligned}
$$

subject to the constraint $\int_{0}^{1} \eta(z) d z=1$, and with

(a) $h=\bar{f} \bar{M} H D^{2} / K$ the dimensionless magnetic field;

(b) $w=\bar{f} W_{p} D^{2} / K$ the dimensionless coupling ( $W_{p}$ now per unit volume) between the nematic and magnetic orientations; we refer to this quantity as the ferronematic coupling parameter; and

(c) $t=k_{B} T \bar{f} D^{2} /(\nu K)$ the dimensionless temperature; this is roughly the ratio of the thermal energy of the colloidal particles to the nematic elastic free energy density.

We note that this nondimensionalization scheme differs from that in a number of previous papers $[10,11,13,14]$. In the previous normalization scheme, it turns out not to be possible to remove the magnetic segregation in a regular way. By contrast, the scheme introduced in our previous letter [15] and further developed here permits a simple limit in which there is no magnetic segregation. This corresponds to an infinite temperature limit with respect to the energy parameter driving the segregation. Segregation can then be considered as a finite temperature perturbation. The natural perturbation parameter is then a nondimensionalized inverse temperature.

Suitable surrogates for the global behaviors of the parameters will be the quantities $\theta_{0}(h)=\theta\left(h, z=\frac{1}{2}\right)$ and the analogous quantity $\psi_{0}(h)=\psi\left(h, z=\frac{1}{2}\right)$. We shall also define the degree of segregation in a number of ways. The segregation order parameter $s(h)$ is an integral quantity and is defined by

$$
s=-\int_{0}^{1} d z \eta(z) \cos 2 \pi z .
$$

The quantity 


$$
\eta_{0}(h)=\eta\left(h, z=\frac{1}{2}\right)
$$

is in some respects analogous to $\theta_{0}(h)$ and $\psi_{0}(h)$ and measures the ratio of the concentration in the center of the cell to its average value.

3. Unsegregated limit. To begin with we put $t \rightarrow \infty$ [18]. The colloidal concentration responds in a Boltzmann-like fashion to its local potential energy. In the infinite temperature limit, therefore, there is no response; the concentration $\eta(z)$ remains constant, and there is no colloidal segregation. Equation (3) and the resulting field theory are both now much simplified.

The theory now reduces to minimizing

$$
F=\int_{0}^{1} d z\left[\frac{1}{2}\left(\frac{d \theta}{d z}\right)^{2}-h \sin \psi+w \sin ^{2}(\theta-\psi)\right] .
$$

3.1. Infinitely strong ferronematic coupling. Now $w \rightarrow \infty$, which enforces $\theta=\psi$; the nematic director follows the magnetic particle distortion and is always perpendicular to the magnetic particles. The resulting problem is now similar to the classical Frederiks problem but lacks the symmetry-breaking characteristic of this problem. The theory now reduces to

$$
F=\int_{0}^{1} d z\left[\frac{1}{2}\left(\frac{d \theta}{d z}\right)^{2}-h \sin \theta\right] .
$$

The Euler-Lagrange equation is

$$
\frac{d^{2} \theta}{d z^{2}}+h \cos \theta=0
$$

The weak field solution can be calculated by putting $\cos \theta=1$ in this equation. Substituting $\theta(0)=\theta(1)=0$ then yields

$$
\theta(z)=\frac{h}{2} z(1-z)
$$

One suitable figure of merit for the total degree of reorientation is $\Theta=\int_{0}^{1} \theta(z) d z$. At low fields $\Theta=h / 12$; the effect is proportional to the imposed field and is thresholdless. Alternatively, we may choose $\theta_{0}(h)=\theta\left(h, z=\frac{1}{2}\right)$. From (9), we find $\theta_{0}(h)=\frac{h}{8}$. Later in this paper we shall find it useful to expand $\theta(h, z) \approx \theta_{0}(h) \sin \pi z$ in a harmonic approximation. If we minimize the linearized version of (7) within this approximation, we obtain $\theta_{0}(h) \approx\left(4 / \pi^{3}\right) h \approx 0.129 h$, rather close to the $\theta_{0}(h)=0.125 h$, given by $(9)$.

A strong field solution can be found using a matched asymptotic expansion method [19]. A high field is here defined by $h \geq 1$ in the absence of any other scale on which to compare it. We omit the details, as only the conclusion is important for the subsequent discussion. There is a boundary region close to the wall, in which the distortion is small but rapidly increases:

$$
\theta(z) \approx 1.46 h^{1 / 2} z-0.5\left(h^{1 / 2} z\right)^{2} .
$$

This region has thickness $z_{0}=0.46 h^{-1 / 2}$. In the bulk of the cell, the solution for $\theta$ is approximately given by

$$
\theta(z)=\frac{\pi}{2}-3.16 \exp \left[-\frac{h^{1 / 2}}{2}\right] \cosh \left[h^{1 / 2}\left(z-\frac{1}{2}\right)\right] .
$$


The key result is that the director distortion saturates. There are boundary regions close to the walls in which the distortion reduces to zero of thickness $z_{0} \sim h^{-1 / 2}$, which become increasingly thin with increasing field. The value of the distortion in the center of the cell becomes increasingly close to saturation, with

$$
\theta_{0}(h)=\frac{\pi}{2}-3.16 \exp \left[-\frac{h^{1 / 2}}{2}\right]
$$

and

$$
\frac{\pi}{2}-\Theta(h) \sim h^{-1 / 2} .
$$

We note that all figures of merit for the total degree of reorientation, including the optical phase lag measured through the cell, monotonically increase with magnetic field up to saturation, although this increase does of course slow at high fields.

These results do not, however, carry over exactly into the finite coupling case. This is because the degree to which the saturated alignment of the magnetic director is limited by the degree of coupling between the nematic and the magnetic directors, as we shall see below.

3.2. Finite ferronematic coupling. This corresponds to the more realistic case of finite anchoring at the colloidal particle surface. The appropriate free energy functional is given by (6):

$$
F=\int_{0}^{1} d z\left[\frac{1}{2}\left(\frac{d \theta}{d z}\right)^{2}-h \sin \psi+w \sin ^{2}(\theta-\psi)\right] .
$$

The Euler-Lagrange equations corresponding to the free energy (6) are

$$
\begin{aligned}
\frac{d^{2} \theta}{d z^{2}}-w \sin (2(\theta-\psi)) & =0, \\
h \cos \psi+w \sin (2(\theta-\psi)) & =0 .
\end{aligned}
$$

Equation (14b) is the bonding equation of Burylov and Raikher [10, 11]. It is apparent that so long as the ratio $\frac{h}{w} \ll 1$, we can expect that $\theta \approx \psi$. The magnetic and nematic directors will be coaligned (or bonded) in this circumstance. If this condition does not hold, then $\theta \approx \psi$ may still hold, but only if $\cos \psi$ is sufficiently small (i.e., $\psi$ is sufficiently close to $\frac{\pi}{2}$ ). Much of this paper is concerned with determining the details of when and in what way this relation holds. It ceases to hold with increasing field when $h$ and $w$ are of the same order of magnitude. The magnetic director becomes more aligned with the field, but the nematic director is no longer aligned with it. This phenomenon has been described as decoupling by Burylov et al. [13] .

3.2.1. Boundary conditions. From a mathematical point of view these equations are slightly peculiar. The strong surface anchoring condition on $\theta$ yields $\theta(0)=$ $\theta(1)=\theta_{s}=0$. However, the boundary conditions $\psi(0)=\psi(1)=\psi_{s}$ are not defined explicitly. However, they are implicit in (14b) from the surface condition $\theta=0$. This yields

$$
h \cos \psi_{s}=w \sin \left(2 \psi_{s}\right)
$$

which sustains two solutions: 


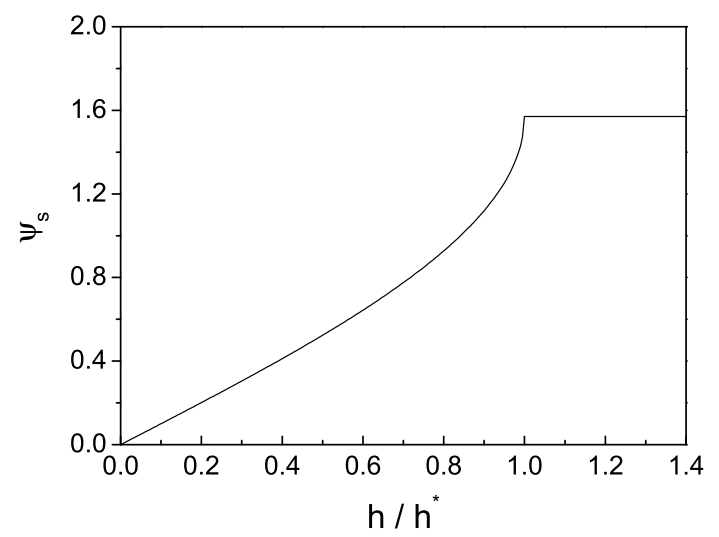

FIG. 2. Behavior of the displacement of the magnetic director at the surface $\psi_{s}$, as a function of scaled magnetic field $h$. Note the linear behavior at low $h$, and the sharp singularity at $h=h^{*}$, at which the $\psi_{s}$ abruptly saturates.

(a) $\psi_{s}=\arcsin (h / 2 w)$, which is the low magnetic field case. For $h / 2 w>1$, however, $\psi_{s}=\arcsin (h / 2 w)$ is no longer well defined.

(b) $\psi_{s}=\pi / 2$, which is the high magnetic field case. The surface value of the magnetic angle $\psi_{s}$ saturates.

Thus $\psi_{s}(h)$ is a monotonic function of $h$, increasing smoothly from zero at $h=0$. For low $h, \psi_{s} \sim h$; the behavior is linear. But there is a change of regime at $h=$ $h^{*}(w)=2 w$, at which point $\psi_{s}=\psi(0)=\psi(1)$ saturates. The behavior of $\psi_{s}$ is shown in Figure 2. The quantity $\psi_{s}$ saturates abruptly at $h=h^{*}$. There is strongly discontinuous behavior in the gradient $\frac{d \psi_{s}}{d h}$ at $h=h^{*}$. Just below $h=h^{*}$, we have

$$
\psi_{s} \sim \frac{\pi}{2}-\sqrt{2}\left(1-\frac{h}{h^{*}}\right)^{1 / 2} .
$$

Given the coupling between $\psi$ and $\theta$, one might also expect some nonanalytic properties in $\psi(z)$ (and hence in $\theta(z)$ ) for all $z$ as a function of $h$. Numerical evidence suggests, however, that the singularity is rounded away from the boundary. We shall return to this problem elsewhere.

3.2.2. Low field properties. We can now expand (6) in powers of small quantities $h, \theta_{0}$, and $\gamma_{0}$, where

$$
\theta(z) \approx \theta_{0} \sin \pi z ; \quad \psi(z)=\psi_{s}+\gamma_{0} \sin \pi z ; \quad \psi_{s}=\arcsin \left(\frac{h}{2 w}\right) .
$$

The free energy is then given by

$$
\begin{aligned}
F= & \int_{0}^{1}\left\{\frac{\pi^{2}}{2} \theta_{0}^{2} \cos ^{2} \pi z-h \sin \left[\arcsin \frac{h}{2 w}+\gamma_{0} \sin \pi z\right]\right. \\
& \left.+w \sin ^{2}\left[\arcsin \frac{h}{2 w}+\left(\gamma_{0}-\theta_{0}\right) \sin \pi z\right]\right\} d z .
\end{aligned}
$$

We now expand the integrand of (18) in a multivariate Taylor series up to second order in $\theta_{0}$ and $\gamma_{0}$, noting that $\sin \left(\arccos \frac{h}{2 w}\right)=\cos \left(\arcsin \frac{h}{2 w}\right)=\sqrt{1-\frac{h^{2}}{4 w^{2}}}$. Performing the integrals, including those over $\sin \pi z$ and $\sin ^{2} \pi z$, we obtain the following 
expansion in $\theta_{0}$ and $\gamma_{0}$ :

(19)

$F=-\frac{h^{2}}{4 w}-\frac{2 h}{\pi} \sqrt{1-\frac{h^{2}}{4 w^{2}}} \theta_{0}+\frac{\pi^{2}}{4} \theta_{0}^{2}+\frac{h^{2}}{8 w} \gamma_{0}^{2}+\frac{w}{2}\left(1-\frac{h^{2}}{2 w^{2}}\right)\left(\theta_{0}-\gamma_{0}\right)^{2}+O\left(\theta_{0}^{3}, \gamma_{0}^{3}\right)$.

This leads to Lagrange equations

$$
\begin{aligned}
\frac{\pi^{2}}{2} \theta_{0}+w\left(1-\frac{h^{2}}{2 w^{2}}\right)\left(\theta_{0}-\gamma_{0}\right)-\frac{2 h}{\pi} \sqrt{1-\frac{h^{2}}{4 w^{2}}} & =0, \\
\frac{h^{2}}{4 w} \gamma_{0}-w\left(1-\frac{h^{2}}{2 w^{2}}\right)\left(\theta_{0}-\gamma_{0}\right) & =0 .
\end{aligned}
$$

Solving these we find

$$
\theta_{0}=\frac{16 h w^{3}}{\pi\left(4 w^{3} \pi^{2}+2 w^{2} h^{2}-\pi^{2} h^{2} w-h^{4}\right)}\left(1-\frac{h^{2}}{4 w^{2}}\right)^{3 / 2}=\frac{4 h}{\pi^{3}}-\frac{\pi^{2}+4 w}{2 \pi^{5} w^{2}} h^{3}+O\left(h^{5}\right)
$$

and

$$
\theta_{0}-\gamma_{0}=\frac{h^{3}}{\pi^{3} w^{2}}+O\left(h^{5}\right)
$$

In the limit $\frac{h}{w} \rightarrow 0$, as expected, this solution tends to the behavior of the infinitely strong-coupling regime (9). In the very low field regime, the response is as though the torque is acting directly on the nematic director. But this response is modified by a third order term in $h$. This reduces the response as compared to the infinite coupling case, as one might expect, given that the restoring force on the nematic director acts only through the intermediary effect of the magnetic director.

3.2.3. Infinite field limiting properties. For sufficiently high fields, the minimizer of the free energy (6) with respect to $\psi$ requires that $\psi(z)=\frac{\pi}{2}$ everywhere. Now (6) reduces to

$$
F \sim \int_{0}^{1} d z\left[\frac{1}{2}\left(\frac{d \theta}{d z}\right)^{2}+w \sin ^{2}\left(\theta-\frac{\pi}{2}\right)\right] \sim \int_{0}^{1} d z\left[\frac{1}{2}\left(\frac{d \theta}{d z}\right)^{2}-w \sin ^{2} \theta\right]
$$

This is just the classic Frederiks transition problem, whose solution is well known [20].

The nematic distortion now no longer saturates at high fields. Indeed, for $w \leq$ $w_{c}=\frac{\pi^{2}}{2}, \psi(z)=\frac{\pi}{2}$ everywhere; i.e., the magnetic distortion is maximal. But apparently paradoxically the cost of any nematic distortion is positive, and hence $\theta(z) \equiv 0$; the nematic distortion is minimal.

However, as $w$ increases beyond $w_{c}$, the high field nematic distortion increases. In the region $w \sim w_{c}$, we can again make the approximation $\theta(z)=\theta_{0} \sin \pi z$, in which case (23) reduces to

$$
F\left(\theta_{0}\right) \sim \frac{1}{2}\left(\frac{\pi^{2}}{2}-w\right) \theta_{0}^{2}+\frac{w}{8} \theta_{0}^{4}+\ldots
$$

Minimizing this with respect to $\theta_{0}$ yields

$$
\theta_{0} \approx \sqrt{2}\left(1-\frac{w_{c}}{w}\right)^{\frac{1}{2}}
$$

Finally for $w \gg w_{c}, \theta(z) \approx \frac{\pi}{2}$ almost everywhere. There is a small healing region close to the boundaries, of dimension $w^{-\frac{1}{2}}$, over which $\theta(z)$ goes from zero to $\frac{\pi}{2}$. 
3.2.4. General classification. On the basis of the evidence adduced above, we are now in a position to make a general classification of $\mathrm{FN}$ behavior into three regimes. The regimes are as follows.

(a) Weak ferronematic coupling. The weak coupling regime is defined by $w \leq$ $w_{c}=\frac{\pi^{2}}{2}$. In this regime, as we have shown in section 3.2 .2 , the initial response $\theta_{0}(h)$ is proportional to $h$. We have shown in section 3.2.3 that $\lim _{h \rightarrow \infty} \theta_{0} \equiv 0$, but in fact the condition is stronger. It will turn out that there is a critical field $h_{c}(w)$ at which the nematic distortion abruptly disappears, so that for $h \geq h_{c}(w), \theta_{0}(h) \equiv 0$. In any event, there is a turning point at intermediate $h_{M}$, and for $h>h_{M}, \theta_{0}(h)$ decreases.

(b) Intermediate ferronematic coupling. We know from section 3.2.2 that at low fields the nematic response increases, with $\theta_{0} \sim h$. If $w>w_{c}$, but $\left|\left(\frac{w}{w_{c}}\right)-1\right| \ll 1$, we also know from section 3.2.3 that $\theta_{0}(h \rightarrow \infty)$ is small. Thus there will be a regime $w_{c}<w<w_{c 2}$ for which necessarily $\theta_{0}(h)$ reaches a maximum as a function of $h$ before decreasing at high fields. The precise value of $w_{c 2}$ remains to be determined.

(c) Strong ferronematic coupling. We know from section 3.1 that in the infinite coupling regime $\theta_{0}(h)$ increases for all $h$. We thus expect a regime defined by $w>w_{c 2}$ for which this behavior is retained.

3.2.5. High field limit. We can now extend the considerations of the infinite field limit to fields which are merely high. In this case, given the considerations of section 3.2.1, this means $h \geq 2 w$. The analysis involves constructing a free energy expansion in both nematic and magnetic orientation variables. Specifically, the expansion extends the considerations of (24) involving $\theta$ to include departures in $\psi$ from complete alignment.

We recall from section 3.2.1 that for large $h, \psi_{s}=\frac{\pi}{2}$. Hence $\psi(z)=\frac{\pi}{2}-\gamma(z)$, where the angle $\gamma(z)$ is small and zero at the boundary. Likewise, at least in the weak and intermediate ferronematic coupling regimes discussed in section 3.2.4, the angle $\theta(z)$ may be regarded as small.

The appropriate expansion variables are then $\gamma_{0}, \theta_{0}$, where

$$
\psi(z) \approx \frac{\pi}{2}-\gamma(z), \quad \gamma(z)=\gamma_{0} \sin \pi z, \quad \theta=\theta_{0} \sin \pi z .
$$

We shall construct a Landau expansion of $F$ in the high field regime. From (6), we obtain

$$
F=F_{0}+\int_{0}^{1} d z\left[\frac{1}{2}\left(\frac{d \theta}{d z}\right)^{2}-h \cos \gamma-w \sin ^{2}(\theta+\gamma)\right],
$$

where $F_{0}$ is a reference free energy defined for $\theta(z) \equiv 0$ and $\psi \equiv \frac{\pi}{2}$. Expanding (27) to fourth order in the angular variables $\theta_{0}, \gamma_{0}$, using the relations (26), yields

$$
F-F_{0}=\left[\frac{\pi^{2}}{4} \theta_{0}^{2}+\frac{h}{4} \gamma_{0}^{2}-\frac{1}{2} w\left(\theta_{0}+\gamma_{0}\right)^{2}\right]-\frac{h}{64} \gamma_{0}^{4}+\frac{1}{8} w\left(\theta_{0}+\gamma_{0}\right)^{4}+\ldots
$$

Here we have isolated the crucial terms quadratic in the variables $\theta_{0}, \gamma_{0}$.

$$
\text { Stability is defined by this quadratic term, which can be written as }
$$

$$
F_{Q}=\frac{1}{2}\left[w_{c} \theta_{0}^{2}+\frac{h}{2} \gamma_{0}^{2}-w\left(\theta_{0}+\gamma_{0}\right)^{2}\right]=\frac{1}{2}\left[\left(w_{c}-w\right) \theta_{0}^{2}+\left(\frac{h}{2}-w\right) \gamma_{0}^{2}-2 w \theta_{0} \gamma_{0}\right],
$$


where we have substituted $w_{c}=\frac{\pi^{2}}{2}$. We have seen in section 3.2.3 that this is the critical ferronematic coupling beyond which the infinite field nematic distortion no longer vanishes, i.e., $\lim _{h \rightarrow \infty} \theta_{0} \neq 0$. And indeed, when $\gamma_{0} \equiv 0$, then by inspection it is clear that the expression given in (29) is positive definite (and hence $\theta_{0} \equiv 0$ ) if and only if $w<w_{c}$.

For finite $h, \theta_{0}=\gamma_{0}=0$ are minimizers of the free energy expression (28) if and only if the quadratic term (29) is positive definite. This will be the case if

$$
w_{c}-w>0
$$

and also if the discriminant of expression (29) is positive:

$$
\left(\frac{h}{2}-w\right)\left(w_{c}-w\right)>w^{2}
$$

Rearranging (30b), we obtain a condition

$$
\frac{h}{2}\left(w_{c}-w\right)-w w_{c}>0
$$

or

$$
h^{-1}<h_{c}^{-1}(w)=\frac{1}{2}\left(w^{-1}-w_{c}^{-1}\right) .
$$

The result of this calculation is that in the weak FN coupling limit, i.e., if $w-w_{c}<0$, then the magnetic director is completely saturated, the nematic distortion will be zero not only in the limit of infinitely high field but also for all fields $h>h_{c}(w)$, where

$$
h_{c}(w)=\frac{2}{\left(w^{-1}-w_{c}^{-1}\right)}=\frac{2 w w_{c}}{\left(w_{c}-w\right)} .
$$

Equivalently, the quadratic form (29) is positive definite if both $w_{c}-w>0$ and $h-h_{c}>0$. Using (29), the free energy (28) can then be recast in a diagonal form in which the change of character at $h=h_{c}, w=w_{c}$ becomes explicit:

$$
\begin{aligned}
F-F_{0}=\frac{1}{2}\left[\frac{2 w_{c}^{2}}{2 w_{c}+h}\left(\theta_{0}-\frac{h}{2 w_{c}} \gamma_{0}\right)^{2}\right. & +\frac{\left(h-h_{c}(w)\right)\left(w_{c}-w\right)}{2 w_{c}+h}\left(\theta_{0}+\gamma_{0}\right)^{2} \\
& \left.+\frac{w}{4}\left(\theta_{0}+\gamma_{0}\right)^{4}-\frac{h}{32} \gamma_{0}^{4}\right]
\end{aligned}
$$

In all cases of interest there exists a minimizer of $F\left(\theta_{0}, \gamma_{0}\right)$ such that $\theta_{0}, \gamma_{0}$ are either zero or small. The $\gamma_{0}^{4}$ term with a negative coefficient is swamped by the $\left(\gamma_{0}+\theta_{0}\right)^{4}$ term.

3.2.6. The weak coupling regime. This is the regime $w<w_{c}=\frac{\pi^{2}}{2}$. We have seen that the nematic response increases rapidly at low fields and reaches a maximum. $\theta_{0}$ then decreases, reaching zero at $h_{c}(w)$, where from (31b)

$$
\frac{2}{h_{c}(w)}=\frac{1}{w}-\frac{1}{w_{c}}
$$

Copyright $@$ by SIAM. Unauthorized reproduction of this article is prohibited. 
We describe this transition as an inverse Frederiks transition, because at high fields the nematic director remains undistorted, whereas for lower fields, deviation from the zero field equilibrium occurs. We note also that

$$
\lim _{w \rightarrow w_{c}} h_{c}^{-1}(w)=0 ; \quad \frac{h_{c}(w)}{2 w}=\frac{1}{\left(1-w / w_{c}\right)} .
$$

The critical field $h_{c}(w)$ diverges as $w \rightarrow w_{c}$, so that for $w>w_{c}$, the undistorted state no longer exists.

For $h>h_{c}(w)$ the magnetic director $\hat{\mathbf{m}}$ is completely aligned with the magnetic field, corresponding to $\psi(z)=\pi / 2$ everywhere. This magnetic alignment is coupled to the nematic director through the colloidal particles, giving an effective perpendicular field on the nematic of magnitude $w$. As we have seen above, there is a strong analogy with the conventional Frederiks effect. If $w$ is too low, even at high fields the effective aligning force on the nematic particles cannot overcome the elastic energy of the nematic director. The director thus remains unmoved by the field. The critical value $w_{c}$ is just that field which corresponds to the Frederiks transition.

We also note that the alignment of the magnetic director $\psi_{s}$ at the surface saturates at $h^{*}(w)=2 w$. However, in the bulk the magnetic saturation occurs only at the higher field $h_{c}(w)=2 w\left(1-w / w_{c}\right)^{-1}=h^{*}(w)\left(1-w / w_{c}\right)^{-1}$. The effect of the surface singularities in the response of the bulk system close to $h^{*}(w)$ is not clear; preliminary evidence suggests that they may be smoothed out. Cells with weaker nematic-magnetic coupling saturate at lower fields (i.e., more easily) because the saturation is discouraged by the nematic elastic term.

At $h_{c}(w)$ there is a transition to nonzero values of $\theta$ because the magnetic field is no longer sufficiently strong to hold the magnetic director perpendicular to the walls. The magnetic director then orients at some angle to the walls, and this in turn breaks the left-right symmetry to which the nematic director is subject. The nematic director thus follows the distorting magnetic director.

In order to analyze the behavior of $\psi$ and $\theta$ just below $h_{c}$, it is necessary to analyze (33). The quadratic form in this equation is diagonal. The coefficient of the term

$$
\frac{2 w_{c}^{2}}{2 w_{c}+h}\left(\theta_{0}-\frac{h}{2 w_{c}} \gamma_{0}\right)^{2}
$$

is always positive. Thus, apart from corrections, $\theta(z)=\frac{h}{2 w_{c}} \gamma(z)$, or

$$
\gamma_{0}=\frac{2 w_{c}}{h} \theta_{0}
$$

We can now rewrite (33) as an expansion in $\theta_{0}$ alone. In the spirit of Landau theory, close to $h=h_{c}(w)$, we replace all values of $h$ by $h_{c}(w)$ except where the relevant term is $\left(h-h_{c}(w)\right)$. After some algebra, we obtain

$$
F-F_{0}=\frac{w_{c}^{2}}{2 w}\left[\left(1-\frac{h_{c}(w)}{h}\right)\left(1-\frac{w}{w_{c}}\right) \theta_{0}^{2}+\frac{1}{4}\left(\frac{w_{c}}{w}\right)^{2}\left(1-\frac{1}{4}\left(1-\frac{w}{w_{c}}\right)^{3}\right) \theta_{0}^{4}\right] .
$$

We note that the coefficient of the quartic term is always positive, despite the apparent negative term in $\gamma_{0}^{4}$ in (28). Minimizing (36) with respect to $\theta_{0}$ yields

$$
\theta_{0}=\sqrt{2} \frac{w}{w_{c}}\left(\frac{\left(1-w / w_{c}\right)}{\left[1-\frac{1}{4}\left(1-w / w_{c}\right)^{3}\right]}\right)^{1 / 2}\left[\frac{h_{c}(w)}{h}-1\right]^{1 / 2}
$$


exhibiting the expected square root singularity as function of the field. The transition thus follows the normal paradigm.

As the magnetic field is further reduced, the nematic response increases. However, at very low fields, the magnetic director responds little to the magnetic field. Then finally the nematic response also decreases, in sympathy. The qualitative explanation of this phenomenon is as follows. The degree of distortion is associated with the couple exerted on the nematic director. This is proportional to $\sin 2(\theta-\psi)$. For the undistorted nematic $(\theta=0)$ this has a maximum at $\psi=\pi / 4$. We thus expect a maximum in $\theta_{0}=\theta(z=1 / 2)$ to occur when $\psi \approx \pi / 4$.

3.2.7. The intermediate coupling regime. We now discuss the regime for which $\theta_{0}(h)$ is monotonically decreasing at high $h$, but $\lim _{h \rightarrow \infty} \theta_{0} \neq 0$. Now the ferronematic coupling is somewhat larger than in the previous case: $w_{c}<w<w_{c 2}$, where we shall determine $w_{c 2}$.

The Landau expansion again uses (35) connecting $\gamma_{0}$ and $\theta_{0}$. Re-expanding in terms of the one relevant variable $\theta_{0}$ yields

$$
F \sim w_{c}\left(1-\frac{w}{w_{c}}-\frac{2 w}{h}\right)\left(1+\frac{2 w_{c}}{h}\right) \theta_{0}^{2}+\frac{w}{4}\left(1+\frac{2 w_{c}}{h}\right)^{4} \theta_{0}^{4},
$$

where now the $\gamma_{0}^{4}$ term in (33) is negligibly small at $w \gg \pi^{2} / 6$. Minimizing this function, we obtain the following expression for $\theta_{0}(h)$ :

$$
\theta_{0}^{2}(h)=2 \frac{\left(1-\frac{w_{c}}{w}+\frac{2 w_{c}}{h}\right)}{\left(1+\frac{2 w_{c}}{h}\right)^{3}} .
$$

We observe that in the limit $h \rightarrow \infty$, this expression is consistent with (37). Indeed, we can expand $\theta_{0}(h)$ in terms of $\theta_{0}(h=\infty)$, yielding

$$
\theta_{0}^{2}(h)=\theta_{0}^{2}(h=\infty) \frac{\left(1+\frac{2 w_{c}}{h\left(1-\frac{w_{c}}{w}\right)}\right)}{\left(1+\frac{2 w_{c}}{h}\right)^{3}} .
$$

Expanding this, and taking square roots, we obtain, now to leading order in $h^{-1}$,

$$
\theta_{0}(h)=\theta_{0}(\infty)\left[1+\left(\frac{w_{c}}{h}\right)\left(\frac{1}{1-\frac{w_{c}}{w}}-3\right)\right]
$$

This is the key result of this section. It shows that for $w$ such that $\frac{1}{1-w_{c} / w}>3$, $\theta_{0}(h)$ for large fields is an increasing function of inverse field and hence a decreasing function of $h$. This condition can be rewritten as

$$
w_{c}<w<w_{c 2}, \quad w_{c 2}=\frac{3}{2} w_{c} .
$$

Equation (42) improves the estimate of $w_{c 2}$ given in our previous paper [15]. 

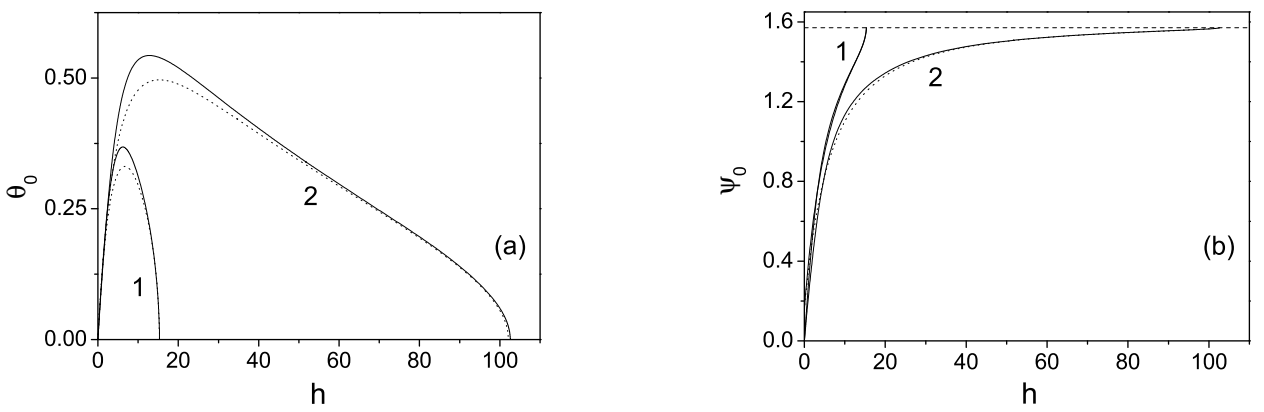

FIG. 3. Weak coupling ferronematics: Nematic (a) and magnetic (b) directors as a function of dimensionless external field, in the absence of a bias field. Quantities $\theta_{0}$ and $\psi_{0}$ in the center of the cell are used as surrogates for global behavior. Curve 1 corresponds to $w=3$ and curve 2 to $w=4.5 ; w<w_{c} \approx 4.93$. The dotted lines have been added to show comparison with the results of the asymptotic calculation (36) in section 3.2.6.

3.2.8. The strong coupling regime. This is the regime $w>w_{c 2}=\frac{3}{2} w_{c}$. We can see from (41) that in this regime, the angle $\theta_{0}$ increases at high field. It is further not clear from the asymptotic analysis whether there is a region of $w$ for which $\theta_{0}$ is not a monotonic function of $h$, or whether the maximum in $\theta_{0}(h)$ disappears exactly at $w_{c 2}$. The numerical results are consistent with the latter hypothesis.

3.3. Numerical results. We have also carried out a numerical minimization of the relevant free energy (3). The method does not involve a direct brute force quadrature-based solution of the resulting Euler-Lagrange equations. Rather we use the existence of a set of first integrals, which allows us to parameterize the solutions in terms of the values of the parameters $\psi_{0}, \theta_{0}, \eta_{0}$, where these quantities are the values of the relevant parameters in the middle of the cell. These quantities satisfy algebraic self-consistency conditions. The method has been used in previous publications [13, 14]. We shall present a detailed discussion of the merits of this approach elsewhere.

Figures 3-6 illustrate the behavior of weak, intermediate, and strong-coupling FNs in the unsegregated limit. Solid curves are numerical solutions. A comparison of numerical calculations and asymptotic results is given by dotted lines.

Figure 3 shows the inverse Frederiks transition. As $h$ increases, the nematic response first increases and then decreases, disappearing at an inverse Frederiks transition at $h=h_{c}(w)$. The magnetic response is a monotonically increasing function of $h$ but saturates at $h=h_{c}(w)$ and $\psi_{0}=\pi / 2$.

The profiles of the nematic and magnetic directors for weak-coupling FNs at low and high fields are shown in Figure 4. These profiles demonstrate that the harmonic approximations for $\theta(z)$ and $\psi(z)$ given in (17) and (26) are extremely good. There is a change of the magnetic director profile shape when $h$ is close to $2 w$. Our investigation shows that this change in the profile structure is due to the ambiguity of the dependence of $\theta(14 \mathrm{~b})$ on $h$ or $\psi$ when $\psi_{s}>\pi / 4$ or $h>\sqrt{2} w$, respectively.

Figure 5 also illustrates peculiar changes in the magnetic director behavior with $h$. For low fields $(h<\sqrt{2} w)$ the magnetic profile is concave-down and $\psi_{0}-\psi_{s}>0$. For high fields the profile is concave-up. In the process of changing the profile from concave-up to concave-down, there is the intermediate concave-convex profile in the narrow interval of $h$ close to $h=2 w$. At a certain value of $h$ in this interval $\psi_{0}=\psi_{s}$ and $\Delta \psi=0$.

Figure 6 shows the nematic and magnetic response in the intermediate and strong- 

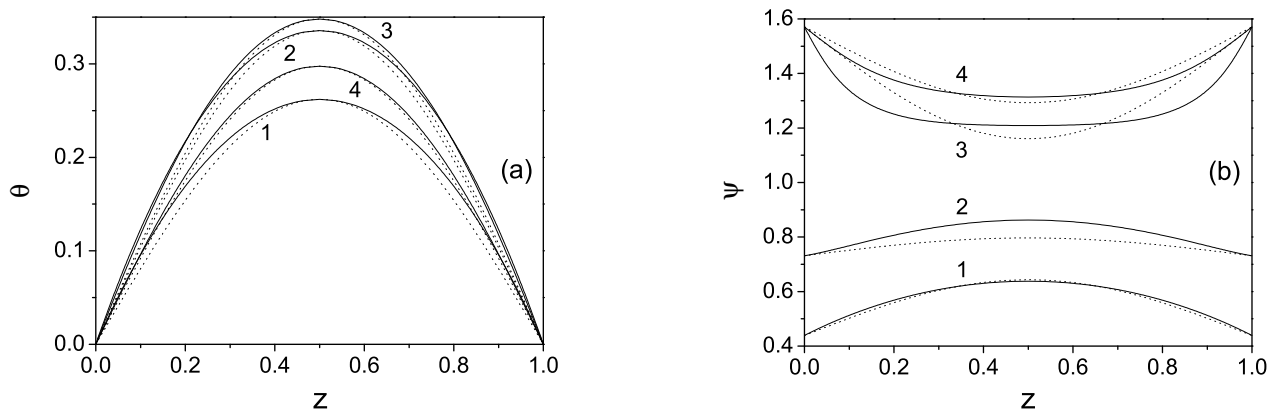

FIG. 4. Weak coupling ferronematics: Nematic distortion $\theta(z)$ (a) and magnetic distortion $\psi(z)$ (b) as a function of position for different dimensionless external fields, in the absence of a bias field, with $w=3, w<w_{c}$. Curve $1, h=2.55$; curve $2, h=4.0$; curve $3, h=8.38$; curve 4 , $h=10.56$. Solid curves are numerical solutions. Dotted curves are asymptotic solutions; in curves 1 and 2 we used the low $h$ expansion, and in curves 3 and 4 we used the high $h$ expansion. Note how $\theta(z)$ always increases in the center of the cell, but in the weak coupling case $\psi(z)$ increases for low $h$ but decreases for higher $h$.

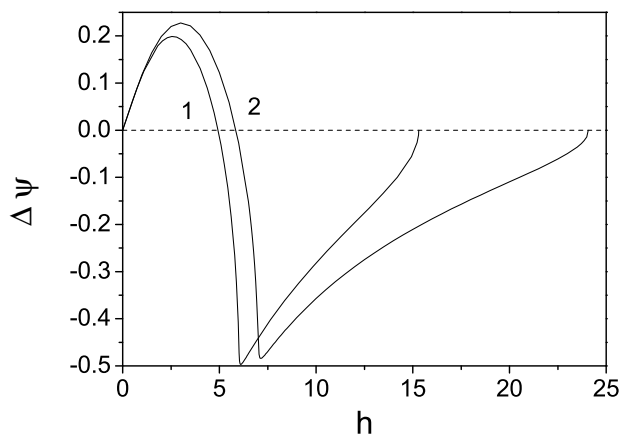

Fig. 5. Differences between surface and bulk behavior as a function of field $h$ for weak ferronematic couplings $w=3$ (curve 1 ) and $w=3.5$ (curve 2). The quantity $\Delta \psi=\psi_{0}-\psi_{s}$ is the difference between the magnetic distortion at the center of the cell and its value at the surface. Note the discussion in the text.

coupling regimes. As $h$ increases, the nematic response of the intermediate coupling FN first increases and then decreases, but $\lim _{h \rightarrow \infty} \theta_{0}=\sqrt{2\left(1-w_{c} / w\right)} \approx 0.6 \neq 0$. In the strong-coupling regime the nematic response is a monotonically increasing function of $h$ and saturates at high $h$. The magnetic response is a monotonically increasing function of the field in both regimes.

4. Bias field. Experimentally, the bias field is imposed in order to maintain particles and hence the director in the $x$ - $z$ plane, whereas we simply assume, even in the absence of a bias field, that the orientation is maintained in this plane. The zerobias-field case then becomes the distinguished limit which we have discussed in the last section. Physically the important point about the bias field is that the resultant magnetic field is never entirely perpendicular to the cell plane. The consequence of this is that the angle $\psi$ can reach its saturation value of $\pi / 2$ only at infinitely high fields (i.e., $\left.h^{-1} \equiv 0\right)$. This contrasts with the zero-bias-field case, for which, as we have seen, at least for $w<w_{c}, \psi \equiv 0$ for $h>h_{c}(w)$.

For analytical purposes it is convenient to discuss small perturbations from the 

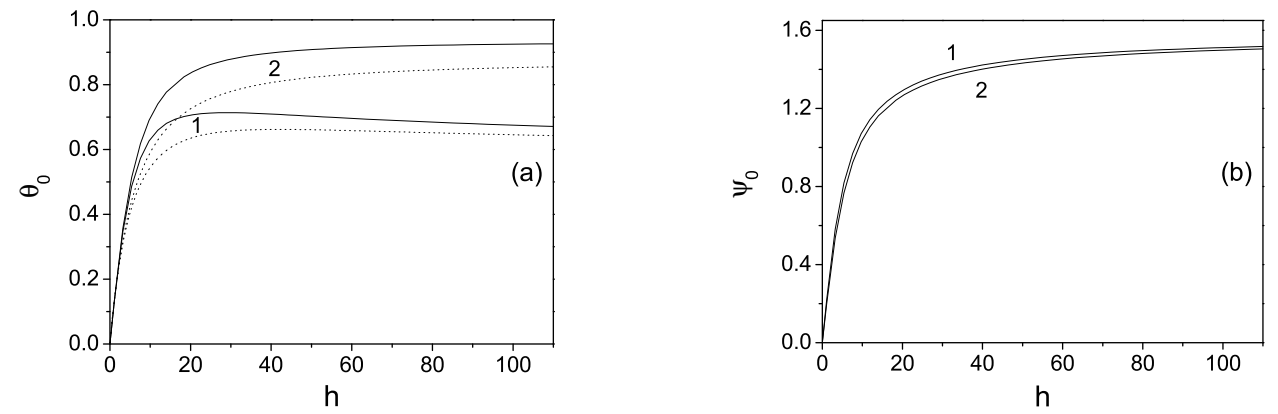

FIG. 6. Intermediate and strong coupling ferronematics: Nematic (a) and magnetic (b) directors as a function of dimensionless external field, in the absence of a bias field. (Curve 1) $w=6$, $w_{c}<w<3 w_{c} / 2$, corresponding to intermediate ferronematic coupling; (curve 2) $w=8, w>3 w_{c} / 2$, corresponding to strong ferronematic coupling. Dotted lines show comparison with the results of the asymptotic calculation which minimizes the free energy (28). This figure is the intermediate and strong FN coupling version of Figure 3.

zero-bias-field case already considered. It is sensible to scale the bias field, by analogy with other nondimensionalization in the problem, leading to a dimensionless bias field $h_{b}=\bar{f} \bar{M} H_{b} D^{2} / K$. The constant $\eta$, high $t$ free energy is now

$$
\begin{aligned}
F= & \int_{0}^{1} d z\left[\frac{1}{2}\left(\frac{d \theta}{d z}\right)^{2}-h \sin \psi-h_{b} \cos \psi\right. \\
& \left.+w \sin ^{2}(\theta-\psi)\right]
\end{aligned}
$$

This is the bias field analogue of (3). The Euler-Lagrange equations now become

$$
\begin{aligned}
\frac{d^{2} \theta}{d z^{2}}-w \sin (2(\theta-\psi)) & =0, \\
h \cos (\psi)-h_{b} \sin \psi+w \sin (2(\theta-\psi)) & =0,
\end{aligned}
$$

where (14a) and (44a) are identical, and (44b) differs from its zero-bias-field analogue (14b) by an extra factor $-h_{b} \sin \psi$.

The most dramatic effects of the bias field occur in the low $w$ regime. In this regime, when there is no bias field, the magnetic director saturates. The saturation drives the high field absence of nematic director distortion and the inverse Frederiks transition. The bias field destroys the magnetic saturation and thus fundamentally affects the inverse Frederiks effect.

We first discuss the boundary values $\psi_{s}$. We recall from section 3.2.1 that in the absence of a bias field, $\psi_{s}(h)$ is a monotonically increasing function of $h$, saturating at $\psi_{s}=\frac{\pi}{2}$ at $h=h^{*}=2 w$. We shall investigate the behavior of $\gamma_{s}=\frac{\pi}{2}-\psi_{s}$, which in the $h_{b}=0$ case vanishes identically for $h>2 w$. Combining (44b) and the condition $\theta_{s}=0$ yields the bias field analogue of (15):

$$
h \cos \psi_{s}-h_{b} \sin \psi_{s}=w \sin 2 \psi_{s} .
$$

For $h_{b}=0$ there are two regimes separated by a singularity at $h=2 w$. In the $h>2 w$ regime $\psi_{s}=\pi / 2 ; \gamma_{s}=0$, whereas for $h<2 w, \gamma_{s} \neq 0$. However, a finite value 


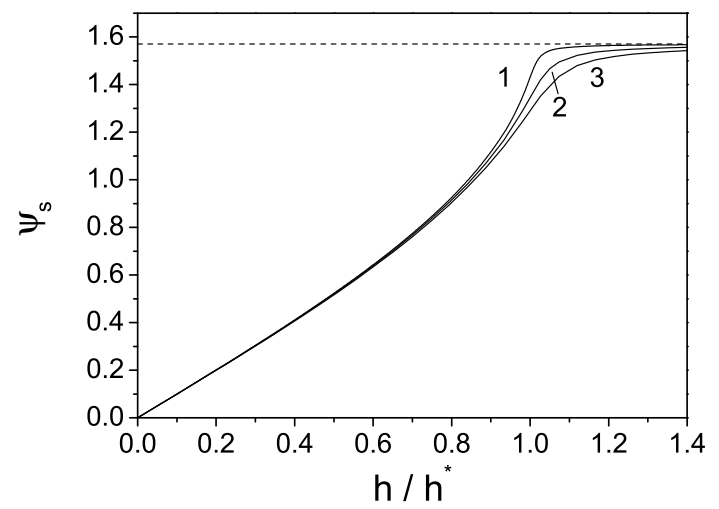

FIG. 7. Behavior of the magnetic surface director deviation $\psi_{s}(h)$ as a function of bulk field $h$ for various different values of bias field $h_{b}$, with $w=0.9, w<w_{c}$. Curve $1: h_{b}=0.01$. Curve 2 : $h_{b}=0.05$. Curve 3: $h_{b}=0.1$. Compare to the zero-bias-field case shown in Figure 2. Note the rounding of the sharp singularity at $h=h^{*}=2 w$.

of $h_{b}$ rounds this singularity, and the behavior of $\psi_{s}$ and hence all other quantities can no longer be divided into two distinct regimes. Rewriting (45) in terms of $\gamma_{s}$, we obtain

$$
h \sin \gamma_{s}-h_{b} \cos \gamma_{s}=w \sin 2 \gamma_{s} .
$$

Linearizing in the regime of small $\gamma_{s} \ll 1$ yields

$$
h \gamma_{s}-h_{b}=2 w \gamma_{s},
$$

yielding

$$
\gamma_{s}=\frac{h_{b}}{h-2 w} .
$$

We plot exact numerical results for $\psi_{s}(h)$ for a number of different values of $h_{b}$ in Figure 7. Equation (48) can be regarded as a response by the FN surface to a bias field probe. The unbiased system is unstable with respect to a perturbation of $\gamma_{s}$ at $h=2 w$. One should thus expect that the susceptibility of the magnetic director to the small bias field would diverge at $h=2 w$, as indeed occurs (although for fields of this order, the small $\gamma_{s}$ approximation no longer holds). The nonzero $\gamma_{s}$ is a signature of a nonzero $\gamma_{0}$, and hence of a nonzero $\theta_{0}$, as we now show.

Analogous results are found for the magnetic and nematic director deviations in the center of the sample. Numerical results are presented in Figure 8.

The results in Figure 8 can be understood semiquantitatively as follows. Using the expansion of (26), we can rewrite the functional (43) in a power law expansion in $\gamma_{0}$ and $\theta_{0}$, yielding for the relevant terms

$$
\begin{array}{r}
F-F_{0}=\frac{1}{2}\left\{\left(w_{c}-w\right)\left[\theta_{0}-\frac{h_{c}(w)}{2 w} \gamma_{0}\right]^{2}+\frac{1}{2}\left(h-h_{c}(w)\right) \gamma_{0}^{2}\right. \\
\left.-\frac{4}{\pi} h_{b} \gamma_{0}+\frac{w}{4}\left(\theta_{0}+\gamma_{0}\right)^{4}-\frac{h}{32} \gamma_{0}^{4}+\frac{4}{9 \pi} h_{b} \gamma_{0}^{3}\right\} .
\end{array}
$$

Copyright $@$ by SIAM. Unauthorized reproduction of this article is prohibited. 

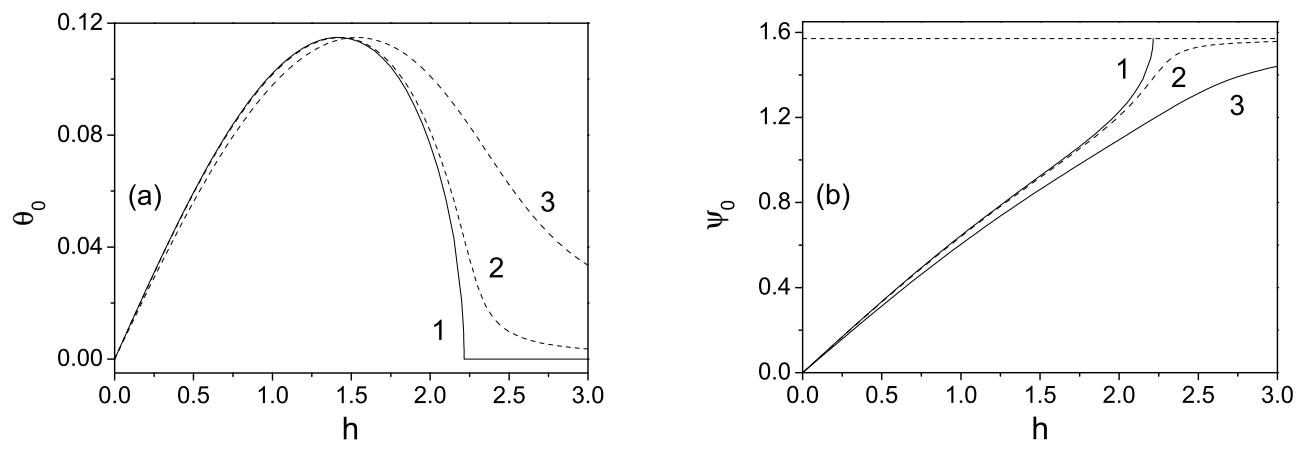

FIG. 8. Effect of bias field on the bulk distortion in the weak ferronematic coupling regime. In all cases $w=0.9, w<w_{c}$, and the nematic director (a) behavior is shown. Curve 1: No bias field $\left(h_{b}=0\right)$. Curves 2 and 3: Weak bias field $\left(h_{b}=0.01,0.1\right)$. The re-entrant Frederiks transition is rounded in the presence of a bias field. The analogous curve for the magnetic director (b) is monotonic as a function of $h$, saturates at $\pi / 2$ for $h_{b}=0$, but merely tends to $\pi / 2$ as $h \rightarrow \infty$ for $h_{b} \neq 0$.

For high fields, the main balance defining $\gamma_{0}$ comes from the linear and quadratic terms; the fourth order terms can be neglected. With this assumption, we minimize (49). This yields $\gamma_{0}=\left(2 w_{c} / h_{c}(w)\right) \theta_{0}$ for the relationship between $\gamma_{0}$ and $\theta_{0}$, as well as an equation for $\gamma_{0}(h)$ :

$$
\gamma_{0}=\frac{4}{\pi\left(h-h_{c}(w)\right)} h_{b}
$$

There is thus no longer an unambiguous distinction between the high field $h>$ $h_{c}(w)$ regime, for which $\gamma=\theta_{0}=0$, and the low field $h<h_{c}(w)$ regime, for which $\gamma_{0} \neq \theta_{0} \neq 0$. In the high field regime both $\theta$ and $\psi$ differ from their zero-bias values 0 and $\pi / 2$, respectively, by quantities which are proportional to the bias field, and appear for $h \gg h_{c}(w)$ to be heading for a divergence at $h=h_{c}^{+}(w)$. This divergence is, however, prevented by higher order terms in (49) which have been neglected here. On the other hand, there is no qualitative change in the low field response; the magnitude of the response is proportional to $h_{b}$.

Taking into account fourth order terms in (49), we find at $h=h_{c}(w)$

$$
\gamma_{0} \approx 4 w\left[\frac{h_{b}}{\pi h_{c}(w)\left(h_{c}^{3}(w)-2 w^{3}\right)}\right]^{1 / 3} .
$$

Here the magnitude of the response is proportional to $h_{b}^{1 / 3}$.

5. Ferroparticle segregation. In the theory as written so far, the ferroparticle density is kept constant. This corresponds to the limit $\eta=1$ in (3), which in turn follows in the infinite temperature limit $t \rightarrow \infty$. However, inserting a finite value of $t$ permits the ferroparticle density to respond so that more particles can migrate to regions where the ferronematic coupling energy is minimized. Interestingly (and apparently paradoxically) the constant $\eta$ limit corresponds to inserting an apparently infinite rather than a zero term. We note that in the real problem $t$ takes its physical value; it is useful nevertheless to treat it as a variable parameter in the theory.

We recall the figures of merit for the degree of segregation $s$ defined in (4) and $\eta_{0}$ defined in (5) in section 2 . Then $s=0,1$ are the limits of complete lack of segregation and segregation, respectively. The quantity $s$ is a segregation order parameter, 
and it possesses a status comparable in the theory to the angular quantities $\theta_{0}$ and $\psi_{0}$. In the low segregation limit we can expand the normalized colloidal density as follows:

$$
\eta(z)=1-2 s \cos 2 \pi z .
$$

5.1. Zero bias field. The full free energy is given by (3):

$$
F=\int_{0}^{1} d z\left[\frac{1}{2}\left(\frac{d \theta}{d z}\right)^{2}+\eta t \ln \eta-\eta h \sin \psi+\eta w \sin ^{2}(\theta-\psi)\right],
$$

subject to the constraint $\int_{0}^{1} \eta(z) d z=1$. The Euler-Lagrange equations are now

$$
\begin{aligned}
\frac{d^{2} \theta}{d z^{2}}-\eta w \sin (2(\theta-\psi)) & =0, \\
h \cos \psi+w \sin (2(\theta-\psi)) & =0, \\
t \ln \eta-\left(h \sin \psi-w \sin ^{2}(\theta-\psi)\right) & =\lambda,
\end{aligned}
$$

where $\lambda$ is a Lagrange parameter which enforces density conservation. Equation (53a) modifies (14a) to the case in which segregation is allowed; (53b) is in fact identical to (14b) and is unchanged by the addition of segregation while (53c) is a new equation for the self-consistent degree of segregation.

The exact solution for $\eta(z)$ comes from inverting (53c) and enforcing the density conservation condition $\bar{\eta}=1$. This solution is

$$
\eta(z)=\frac{\exp \left\{\left[h \sin \psi-w \sin ^{2}(\theta-\psi)\right] / t\right\}}{\int_{0}^{1} d z \exp \left\{\left[h \sin \psi-w \sin ^{2}(\theta-\psi)\right] / t\right\}} .
$$

In general, this solution must be determined self-consistently with solutions for $\psi(z)$ and $\theta(z)$, and the detailed picture is complicated.

However, in the high temperature limit $\eta$ is always small. We can then describe the degree of segregation using the order parameter $s$ and perturb away from the infinite $t$ solution using (4). In this limit

$$
s=-\frac{1}{t} \int_{0}^{1} d z \cos 2 \pi z\left[h \sin \psi-w \sin ^{2}(\theta-\psi)\right],
$$

where values of $\theta$ and $\psi$ are given by the infinite $t$ limit. In general, we expect values of $s(h)$ to peak at intermediate $h$, for it is in these cases that $\theta$ and $\psi$ change most across the cell.

A particularly interesting case occurs for high fields in the low ferronematic coupling $w<w_{c}$ regime. Here it is possible to include the segregation order parameter $s$ in an extended Landau expansion closely analogous to (28). The parameter $s$ couples to the variables $\theta_{0}, \psi_{0}$ in this expansion. We recall (17): in this regime $\psi \approx \frac{\pi}{2}-\gamma_{0} \sin \pi z$; $\theta(z)=\theta_{0} \sin \pi z$.

We obtain the following free energy:

$$
\begin{gathered}
F-F_{0}=\frac{1}{2}\left\{\frac{2 w_{c}^{2}}{2 w_{c}+h}\left(\theta_{0}-\frac{h}{2 w_{c}} \gamma_{0}\right)^{2}+\frac{\left(h-h_{c}(w)\right)\left(w_{c}-w\right)}{2 w_{c}+h}\left(\theta_{0}+\gamma_{0}\right)^{2}\right. \\
\left.+\frac{w}{4}\left(\theta_{0}+\gamma_{0}\right)^{4}-\frac{h}{32} \gamma_{0}^{4}+2 s^{2} t-s\left[w\left(\theta_{0}+\gamma_{0}\right)^{2}-\frac{h}{2} \gamma_{0}^{2}\right]\right\} .
\end{gathered}
$$

Copyright $@$ by SIAM. Unauthorized reproduction of this article is prohibited. 
Equation (56) is (33) modified by some extra terms in $s$. The quadratic term in $s$ comes from the $\eta \ln \eta$ term in the free energy (3). The linear term comes from the evident fact that the colloidal density is coupled linearly both to the nematic-magnetic coupling and to the coupling of the magnetic director with the field.

In the high field $h>h_{c}(w)$ limit there is no structure at all in the infinite $t$ regime, for now $\psi(z)=\pi / 2$ and $\theta(z)=0$. As a result, (54) and (55) show that in this regime $s=0$ and there is no segregation. However, in the low $h$ limit there is structure in $\psi(z), \theta(z)$ and so $\eta(h) \neq 0$ for $h<h_{c}(w)$. As a result, the order parameters $s$ and $\gamma_{0}$ couple in a Landau expansion of the free energy of the system close to $h_{c}(w)$. The coupling is constant, but the stabilizing term in $s$ is proportional to $t$. It is this fact which gives rise to the general result $s \sim t^{-1}$.

However, the linear coupling between $s$ and quadratic terms in the other order parameters does have profound consequences. This is a consequence of the HalperinLubensky-Ma theorem [24]. This theorem states that coupling a critical order parameter to a second noncritical order parameter can under some circumstances drive a continuous phase transition first-order.

To show what happens in this case, we minimize (56). We obtain (35) for the relationship between $\gamma_{0}$ and $\theta_{0}$, and additional equations for $s$ and $\gamma_{0}$ :

$$
s=\frac{h h_{c}}{16 t w_{c}}\left[1+\frac{4 w_{c}^{2}-h h_{c}}{h\left(2 w_{c}+h_{c}\right)}\left(1-\frac{h}{h_{c}}\right)\right] \gamma_{0}^{2},
$$

$$
\gamma_{0}^{2}=8\left\{\left[\frac{\left(2 w_{c}+h\right)^{2}}{2 w_{c}\left(2 w_{c}+h_{c}\right)}-\frac{h}{h_{c}}\right] s+\frac{2 w_{c}+h}{2 w_{c}+h_{c}}\left(1-\frac{h}{h_{c}}\right)\right\}\left[\frac{\left(2 w_{c}+h\right)^{4}}{2 w_{c}^{3}\left(2 w_{c}+h_{c}\right)}-\frac{h}{h_{c}}\right]^{-1} .
$$

Substituting (35) and (57) into (56), we can evaluate the fourth order term in $\gamma_{0}$ at $h=h_{c}(w)$ :

$$
F-F_{0}=\left[\frac{h_{c}^{3}(w)}{2}\left(\frac{1}{w^{3}}-\frac{1}{2 t w_{c}^{2}}\right)-1\right] \frac{h_{c}(w)}{64} \gamma_{0}^{4} .
$$

The key point is that the coupling terms provide a negative definite contribution to the fourth order term. The negative magnitude increases as the coupling (which in this case is the temperature) decreases. Eventually, at a sufficiently low temperature the sign of the $\gamma_{0}^{4}$ term in the Landau expansion close to $h=h_{c}(w)$ changes. A negative $\gamma_{0}^{4}$ term signals that the continuous transition at $h=h_{c}(w)$ becomes first order.

From (59) we find that the tricritical point (i.e., the point at which the continuous phase transition becomes first order) occurs for

$$
t_{c}(w)=\frac{w^{3}}{2 w_{c}^{2}\left(1-\frac{2 w^{3}}{h_{c}^{3}(w)}\right)} \approx \frac{w_{c}}{2}\left(\frac{w}{w_{c}}\right)^{3} .
$$

We can also look at the properties of $\gamma_{0}(h)$ and $s(h)$ below $h=h_{c}(w)$ but above $t=t_{c}(w)$. In this region the transition is still continuous but is approaching tricriticality. By substituting (58) into (57) and expanding $s$ in powers of $\left(h_{c}(w)-h\right)$, we find the segregation order parameter

$$
s=\frac{2 w_{c} t_{c}(w)}{h_{c}^{2}(w)\left(t-t_{c}(w)\right)}\left(h_{c}(w)-h\right)
$$




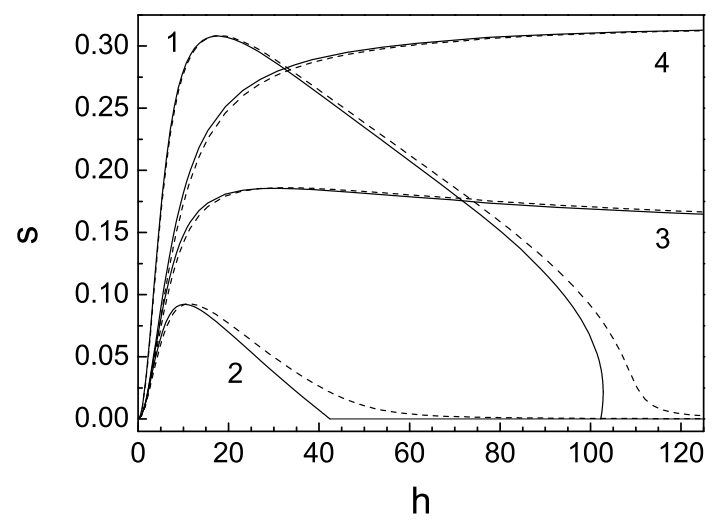

FIG. 9. Behavior of the segregation order parameter as a function of the dimensionless external field in the three ferronematic coupling regimes. (Curve 1) Weak coupling regime; $w=4.5$, $t=1.78<t_{c}=1.87, h_{c}=102.1$. (Curve 2) Weak coupling regime; $w=4, t>t_{c}=1.32$, $h_{c}=42.3$. (Curve 3) Intermediate coupling regime; $w=5.5$. (Curve 4) Strong coupling regime; $w=7$. $t=3.85$ in curves $2-4$. Solid curves: No bias field. Dashed curves: Weak bias field; $h_{b}=0.1$ in curve 1 and $h_{b}=0.4$ in curves $2-4$.

in the lowest order approximation. We find that $s$ goes linearly with $\left(h_{c}(w)-h\right)$ but that the linear coefficient diverges at the tricritical point.

Likewise, from (57) and (58) we find

$$
\gamma_{0}^{2}=\frac{32 t w_{c}^{2} t_{c}(w)}{h_{c}^{4}(w)\left(t-t_{c}(w)\right)}\left(h_{c}(w)-h\right)
$$

Here the characteristic square root behavior for $\gamma_{0}$ is maintained, but the coefficient of proportionality diverges with a square root divergence as the tricritical point is approached.

Beyond the tricritical point (i.e., $t<t_{c}(w)$ ), there are solutions for $h>h_{c}(w)$. These solutions belong to a van der Waals loop. Thus there are two qualitatively different behaviors, separated by a singularity at $t=t_{c}(w)$, with $w<w_{c}$. For $t>t_{c}(w)$ we have the inverse Frederiks transition described in detail in the sections above. For $t<t_{c}(w)$, on the other hand, the functional dependence of $s, \theta$, and $\psi$ develops a van der Waals loop. These cases are illustrated in Figure 9 (solid curves 2 and 1), where we show curves calculated numerically by minimizing the cell free energy (1a).

To show what happens in the intermediate and strong-coupling regimes, we rewrite (56) in the form

$$
\begin{gathered}
F-F_{0}=\frac{1}{2}\left\{\frac{2 w_{c} w}{2 w_{c}+h}\left[\frac{w_{c}}{w}\left(\theta_{0}-\frac{h}{2 w_{c}} \gamma_{0}\right)^{2}-\left(1+h \frac{w-w_{c}}{2 w_{c} w}\right)\left(\theta_{0}+\gamma_{0}\right)^{2}\right]\right. \\
\left.+\frac{w}{4}\left(\theta_{0}+\gamma_{0}\right)^{4}-\frac{h}{32} \gamma_{0}^{4}+2 s^{2} t+s\left[\frac{h}{2} \gamma_{0}^{2}-w\left(\theta_{0}+\gamma_{0}\right)^{2}\right]\right\},
\end{gathered}
$$

where $w>w_{c}$. We now minimize (63). For $t \gg 1$ this yields (35) for the relationship between $\gamma_{0}$ and $\theta_{0}$. The equations for $s$ and $\theta_{0}$ are now 


$$
\begin{gathered}
s=\frac{1}{4 t}\left[w\left(1+\frac{2 w_{c}}{h}\right)^{2}-\frac{2 w_{c}^{2}}{h}\right] \theta_{0}^{2}, \\
\theta_{0}^{2}=\frac{2\left(1-\frac{w_{c}}{w}+\frac{2 w_{c}}{h}\right)\left(1+\frac{2 w_{c}}{h}\right)}{\left(1+\frac{2 w_{c}}{h}\right)^{4}-\frac{2 w_{c}^{4}}{w h^{3}}-\frac{w}{2 t}\left[\left(1+\frac{2 w_{c}}{h}\right)^{2}-\frac{2 w_{c}^{2}}{w h}\right]^{2}} .
\end{gathered}
$$

From (64a) and (64b) one can obtain to lowest order in $h^{-1}$

$$
\begin{gathered}
s=\frac{w-w_{c}}{2 t-w}\left[1+\frac{2 w_{c}^{2}}{w h}\left(\frac{2 w}{w-2 t}-\frac{w_{c}}{w_{c}-w}\right)\right], \\
\theta_{0}^{2}=\frac{4 t}{2 t-w}\left[\frac{w-w_{c}}{w}+\frac{2 w_{c}}{w h}\left(5 w_{c}-2 w+\frac{2 w_{c}\left(2 t-w_{c}\right)}{w-2 t}\right)\right] .
\end{gathered}
$$

Equations (65a) and (65b) are valid in the high field limit if $t>w-w_{c} / 2$ (by definition, $s \leq 1)$. In the limit $t \rightarrow \infty$, (65b) reduces to its no-segregation limit (41). It follows from $(65 \mathrm{~b})$ that the boundary $w_{c 2}$ of the intermediate coupling regime at finite temperature reduces to

$$
w_{c 2}(t)=t+\frac{5}{4} w_{c}-\frac{1}{4}\left(16 t^{2}-8 t w_{c}+9 w_{c}^{2}\right)^{1 / 2} \approx \frac{3}{2} w_{c}\left(1-\frac{w_{c}}{6 t}\right),
$$

and this regime takes place at $w_{c}<w<w_{c 2}(t)$. The strong coupling regime should begin at $w_{c 2}(t)$. It is seen from (64a) that $s(h)$ has a maximum if $w_{c}<w_{s c}(t)$, where

$$
w_{s c}(t)=\frac{w_{c}}{4}\left(1+\sqrt{1+\frac{16 t}{w_{c}}}\right) .
$$

The behavior of the segregation order parameter in the intermediate and strongcoupling regimes is shown in Figure 9 (curves 3 and 4).

5.2. Nonzero bias field. For the nonzero bias field, the free energy can be expressed as

$$
\begin{aligned}
F-F_{0}=\frac{1}{2}\{ & \frac{2 w_{c} w}{2 w_{c}+h}\left[\frac{w_{c}}{w}\left(\theta_{0}-\frac{h}{2 w_{c}} \gamma_{0}\right)^{2}-\left(1+h \frac{w-w_{c}}{2 w_{c} w}\right)\left(\theta_{0}+\gamma_{0}\right)^{2}\right] \\
& -\frac{4 h_{b}}{\pi} \gamma_{0}+\frac{4}{9 \pi} h_{b} \gamma_{0}^{3}-\frac{8 h_{b}}{3 \pi} \gamma_{0} s+\frac{w}{4}\left(\theta_{0}+\gamma_{0}\right)^{4}+2 t s^{2} \\
& \left.-w\left(\theta_{0}+\gamma_{0}\right)^{2} s+\frac{h}{2} \gamma_{0}^{2} s-\frac{h}{32} \gamma_{0}^{4}\right\} .
\end{aligned}
$$

Here (68) is (56) modified by some extra terms in $h_{b}$. Minimizing this equation, we obtain (35) for the relationship between $\gamma_{0}$ and $\theta_{0}$. For high fields we can find the relationship between $s$ and $\gamma_{0}$ :

$$
s=\frac{1}{8 t}\left\{\frac{16}{3 \pi} h_{b} \gamma_{0}-\left[h-2 w\left(1+\frac{h}{2 w_{c}}\right)^{2}\right] \gamma_{0}^{2}\right\} .
$$

Copyright $@$ by SIAM. Unauthorized reproduction of this article is prohibited. 

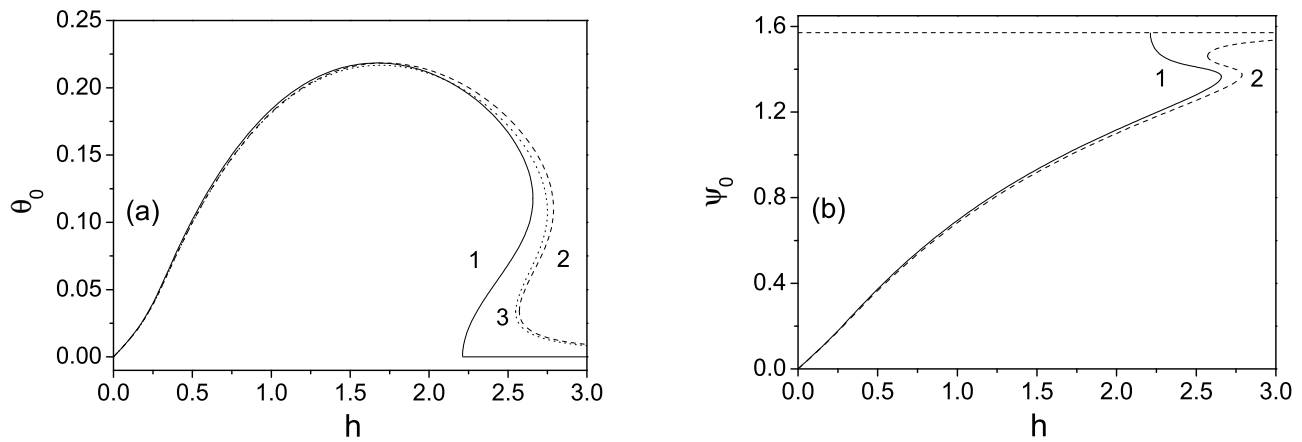

FIG. 10. (a) Director distortion for weak ferronematic coupling at a finite temperature as a function of the external field, showing the development of a van der Waals loop at the inverse Frederiks transition. In both curves $t=0.005, w=0.9\left(t<t_{c} \approx 0.018\right)$. Curve 1: No bias field; $h_{b}=0$. Curve 2: Weak bias field; $h_{b}=0.025$. The analogous curve for the magnetic director (b) is nonmonotonic as a function of $h$, but saturates at $\pi / 2$ for $h_{b}=0$, but merely tends to $\pi / 2$ as $h \rightarrow \infty$ for $h_{b} \neq 0$. Curve 3: The effect of small diamagnetic anisotropy $\left(\kappa=\frac{1}{2} \chi_{a} K /(\bar{f} \bar{M} D)^{2}=0.015\right)$.

A solution for $\theta_{0}$ can be obtained from a cubic equation in which linear and cubic terms play an important role. However, this equation is too complicated and is not presented here.

The dashed curve 2 in Figure 9 shows that the bias field rounds the re-entrant Frederiks transition. The van der Waals loop is retained at the small bias field (not shown in the figure), but no longer occurs at a sufficiently high bias field (dashed curve 1).

We now make remarks concerning the importance of the bias field. The role of the bias field in the $x$ direction is to restrict the nematic and magnetic directors to the $x-z$ plane. In the absence of the bias field but in high applied fields, the system will choose a (broken symmetry) plane in which to orient. A detailed analysis requires a full treatment of the local statistical mechanics of the ferronematic ordering and will be discussed elsewhere.

We have analyzed in detail the behavior of a ferronematic system at high dimensionless temperatures $(t \geq 1)$. However, our analysis is restricted to a weak bias field and runs into difficulties if the magnetic particles are not well aligned. Our segregation parameter $s$ is a useful tool only for high $t$.

In experiments, however, it is more common to encounter a low-coupling regime at low $t$, and it is this regime which is of prime interest for ferronematic applications. However, to investigate the segregation effect we now have to use the quantity $\eta$, which can take values much larger than unity. Furthermore, the asymptotic analysis gives little insight into the system properties at large deviations from the initial alignment. We thus resort to numerical studies. Figures 10-13 show aspects of the system behavior in the low-coupling regime at low temperature $t$ for system parameter values in the experimental region.

In Figures 10 and 11 we illustrate the orientational behavior of the nematic and magnetic directors and the quantity $\eta$ in the varying magnetic field in the middle of the cell. The figures show the development of the van der Waals loop.

We remark that the van der Waals loop, and thus the first order transition between a highly segregated low field phase and a slightly segregated high field case, is retained even when a bias field is introduced. In this case, however, if there is no first order 


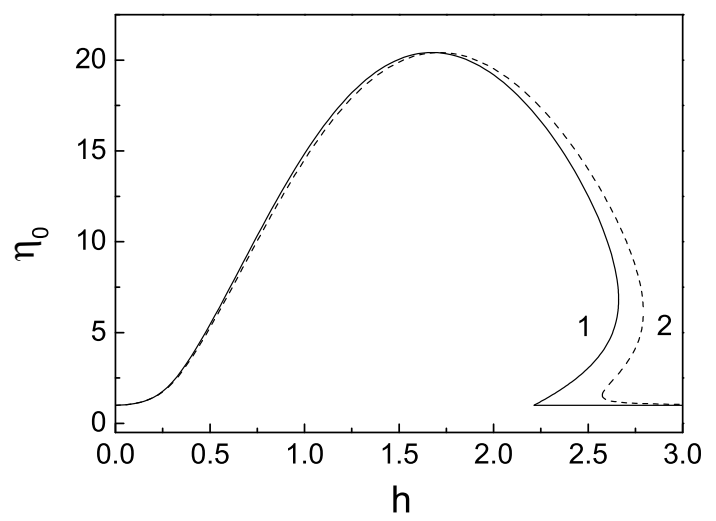

FIG. 11. Ratio of the local to the mean volume particle fraction for weak ferronematic coupling at a finite temperature as a function of the external field, showing the development of a van der Waals loop at the inverse Frederiks transition. In both curves $t=0.005, w=0.9 ; t<t_{c} \approx 0.018$. Curve 1 : No bias field; $h_{b}=0$. Curve 2: Weak bias field; $h_{b}=0.025$.
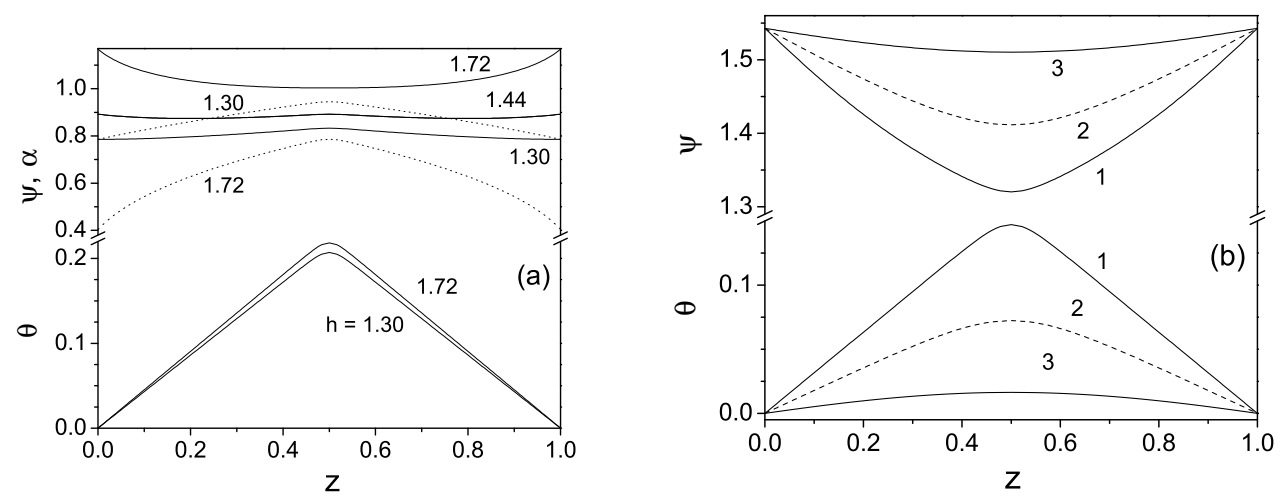

FIG. 12. (a), (b) Orientational profiles at $t=0.005, w=0.9$, and $h_{b}=0.025 . \alpha$ (dotted lines) is the angle between magnetic and nematic directors. Curves 1-3 in (b) correspond, respectively, to the upper, middle (unstable), and lower parts of the van der Waals loop at $h=2.7$.

transition, then the continuous transition found in the zero-bias-field case disappears and is replaced by smooth high field behavior (curve 2 in Figures 10 and 11). In fact, we have found numerical evidence for van der Waals loops in previous calculations [14, 15]. This analysis presented here provides for the first time a consistent explanation.

We also show, in Figures 12 and 13, some examples of the profiles of the nematic and magnetic directors and of magnetic colloid concentration profiles through the cell. In these examples the system is in the weak coupling regime; as the field is increased the system exhibits a van der Waals loop and a consequent first order phase. We recall that in this regime, for low fields, the degree of nematic distortion, represented by $\theta(1 / 2)=\theta_{\max }$, goes through a maximum before tumbling in a discontinuous way and subsequently decreasing to zero in the limit of the high field.

The profile of the magnetic director in Figure 12(a) (given by the angle $\psi$ ) shows a change of regime from low fields to high fields. For low fields the profile is concavedown (i.e., $\left.\psi(1 / 2)>\psi_{s}\right)$, whereas for high fields it is concave-up $\left(\psi(1 / 2)<\psi_{s}\right)$. We 


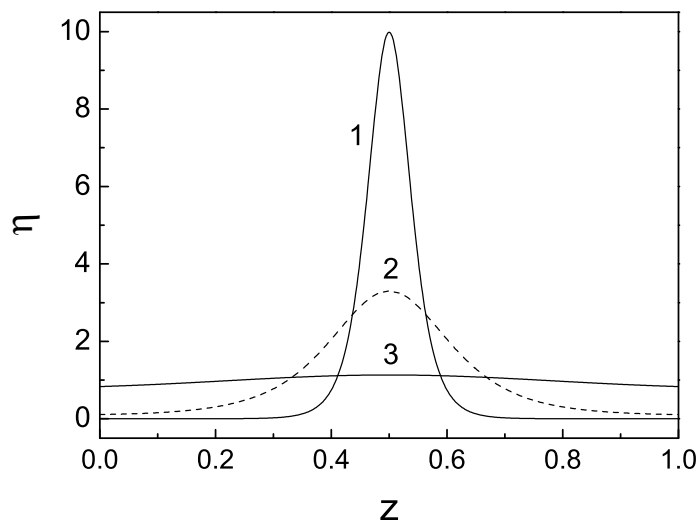

Fig. 13. Concentration profiles at $t=0.005, w=0.9$, and $h=2.7, h_{b}=0.025$. Curves $1-3$ correspond to the upper, middle (unstable), and lower parts of the van der Waals loop, respectively.

have seen this behavior above in the non-bias-field case in Figure 4(b). The change from concave-down to concave-up profiles occurs near the peaks in $\theta_{\max }$ (Figure 10(a)) and $\eta_{\max }$ (Figure 11) $\left(h_{\max }=1.68\right)$. From this figure we note a well-defined intermediate concave-convex profile. The orientational profile of the nematic director $\theta$ is concave-down everywhere as a result of the boundary conditions on $\theta$. However, we note that the behavior of $\theta(z)$ is unlike that in conventional nematics. In conventional nematics the nematic director can saturate over most of the cell apart from a region very close to the boundary. Here, however, we see a constant (absolute) gradient in $\theta$, apart from very close to the center of the cell, where $\theta$ abruptly changes its gradient.

We give here a brief semiquantitative discussion of the changing properties of $\psi(z)$. As $h$ is increased, the magnetic director profile begins to change when $\psi(0)=$ $\psi(1)=\psi_{s}$ reaches the value $\frac{\pi}{4}$. Using (44b) it can be shown that this occurs at $h^{* *}=h_{b}+\sqrt{2} w$. For $h<h^{* *}, \frac{d \psi(0)}{d z}>0$ and $\psi$ increases away from the boundary. At $h^{* *}, \frac{d \psi(0)}{d z}=0$, and for $h>h^{* *}, \frac{d \psi(0)}{d z}<0 ; \psi$ now decreases away from the boundary. There is then a narrow interval in $h$ over which the turning points in $\psi(z)$ move from the edge of the cell toward its center. In this interval for $0<z<z_{c}(h), \frac{d \psi}{d z}<0$, whereas for $z_{c}<z<\frac{1}{2}$, $\frac{d \psi}{d z}>0$, with $\psi(z)=\psi(1-z)$ everywhere. Eventually, for $h>h^{\dagger}$ (which depends in specific cases on $h_{b}$ ), $z_{c}$ reaches $z_{c}=\frac{1}{2}$, and for $h>h^{\dagger}$, $\psi(1 / 2)<\psi_{s}$, and the behavior of $\psi(z)$ is monotonic in the interval $0 \leq \frac{1}{2} \cdot{ }^{1}$

The inverse Frederiks effect can also be interpreted in terms of a force law. The torque of the magnetic particles on the nematic matrix is not a monotonic function of the angle $\alpha=\pi / 2-(\psi-\theta)$ between the directors and reaches its maximum value at $\alpha \approx \pi / 4$.

Finally, in this section, we note that it seems likely that the transition to a ferronematically distorted state actually occurs first through a first order transition at lower fields. Physical values of $t$ are lower than unity; the result is that our analysis remains true in the high field limit but is modified in low field limit. Similarly, we

\footnotetext{
${ }^{1}$ Further computation shows that the change in the profiles $\psi(z)$ is connected with an ambiguity of the dependence $\theta(h, \psi)$ when $\psi_{s}>\pi / 4$ or $h>h_{b}+\sqrt{2} w$. There are two branches, but only one of these satisfies the boundary condition $\theta_{s}=0$. On this branch the angle $\theta$ decreases with $h$ and hence with $\psi$, leading to the inverse Frederiks effect.
} 


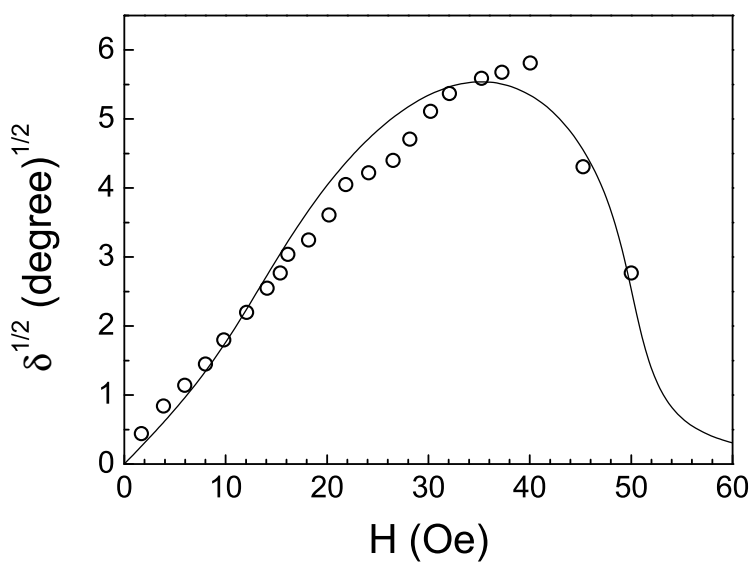

Fig. 14. Experimental data by Chen and Amer [3] of the square root of the phase difference versus the magnetic field strength (open circles) and the theoretical curve obtained by the numerical minimization of the cell free energy equations (1a) and (1b).

find that in dimensionless units the bias field required for the analysis presented here to hold true is $h_{b}<t$. Unsurprisingly, at low temperatures, a small bias field suffices to saturate the local magnetic order.

Figures 12(b) and 13 show the profiles in the magnetic field range where the van der Waals loop is developed. All profiles are symmetrical due to equal nematic anchoring strengths at the walls of the cell.

6. Experimental considerations. To make contact with the experiment $[9,17$, $18,21]$, we consider a cell of thickness $D=125 \mu \mathrm{m}$ and suppose magnetic particles of $L=0.2 \mu \mathrm{m}, d=L / 3$. Plausible mean packing fractions are in the range $\bar{f} \approx$ $2 \times 10^{-7}-6 \times 10^{-5}$, with magnetite particles for which $\bar{M}=485 \mathrm{G}$. The quantity $W_{p} d$ is a quantity with the dimensions of an anchoring energy $W$. Naive microscopic theory suggests that this might be expected to be of the same order of magnitude as the anchoring energy at the surface of the colloidal particle. In this spirit, we expect $W_{p} d \approx 10^{-3}-10^{-1} \mathrm{erg} / \mathrm{cm}^{2}$. Finally, for $5 \mathrm{CB}$ at $T=25^{\circ} \mathrm{C}, K \sim 5.3 \times 10^{-7} \mathrm{dyn}$. The resulting dimensionless quantities are in the range $w \approx 9 \times 10^{-3}-2.7 \times 10^{2}$ and $t \approx 3.5 \times 10^{-3}-1$.

To obtain higher temperatures $t$, in order that real experiments more closely match our ideal systems, it would be necessary to use thicker cells and higher particle concentrations. For example, with a cell of thickness $D=460 \mu \mathrm{m}$, magnetic particles with $L=0.15 \mu \mathrm{m}, d=L / 3$, and a mean volume fraction of $f=6.9 \times 10^{-6}(c=$ $2.3 \times 10^{10} \mathrm{~cm}^{-3}$ ), we have $t=3.85$.

The size and the aspect ratio we consider for the colloidal ferromagnetic particles are consistent with these particles being in a single-domain magnetic state [22]. We suppose that the magnetic grains are coated by suitable surfactant $[3,9,23]$ to prevent coagulation. This theoretical idealization has in practice presented problems for experimentalists, but we do not address these here. Finally, we impose homeotropic boundary conditions at the particle surfaces.

We show in Figure 14 a fit of the experimental data by Chen and Amer [3] to our theory. The field-induced molecular reorientation of the FN was found in [3] by measuring the corresponding induced change in phase difference 


$$
\delta=\frac{2 \pi}{\lambda} \int_{0}^{D}\left[\frac{n_{o} n_{e}}{\left(n_{e}^{2} \cos ^{2} \theta+n_{o}^{2} \sin ^{2} \theta\right)^{1 / 2}}-n_{o}\right] d z,
$$

where $\lambda$ is the wavelength of the incident laser beam and $n_{o}, n_{e}$ are, respectively, the ordinary and extraordinary refractive indices of the sample. The continuous curve is calculated for $\lambda=632.8 \mathrm{~nm}$, the width of an FN cell $D=337 \mu \mathrm{m}, H_{b}=0.6$ Oe, $M_{s}=340 \mathrm{G}\left(\gamma-\mathrm{Fe}_{2} \mathrm{O}_{3}\right.$ magnetic particles $), K_{3}=7.63 \times 10^{-7} \mathrm{dyn}, K_{1}=6 \times 10^{-7} \mathrm{dyn}$, $n_{o}=1.5443, n_{e}=1.7582$, and $\chi_{a}=0.97 \times 10^{-7}\left(\mathrm{MBBA}, T=25^{\circ} \mathrm{C}[17]\right)$. The parameters of the best fit are $L=0.47 \mu \mathrm{m}, L / d=7.7$ (close to that in [3]), $W_{p} d=$ $4.9 \times 10^{-2} \mathrm{erg} / \mathrm{cm}^{2}$, and $\bar{f}=1.83 \times 10^{-8}$. For the above parameter values, $w \approx 0.22$ (low coupling regime). A clear decrease in $\delta$ for $H>40$ Oe is in agreement with our prediction of an inverse Frederiks effect.

We note [10] that the ferronematic is expected to exhibit collective behavior only for colloidal particle concentrations exceeding a critical value $w_{c r}$, with $w_{c r} \sim 1 / 2$. However, our estimate for $w$ is slightly below $1 / 2$. There is thus an apparent paradox that results can be fitted to a model, but only with parameters for which the microscopic foundation of the model is weak. The solution to the paradox is not entirely clear, but it is possible to speculate. Among possible weaknesses in the model are (a) a failure to take account of the polydispersity in size of magnetic particles and (b) the use of a simple phenomenological Rapini-Papoular expression for the anchoring energy [10].

The best fit of the birefringence gives the value of mean particle concentration $\bar{c}$ as about $7 \%$ of the total concentration reported in [3]. We speculate that this may be related to a partial coagulation of the magnetic particles during the preparation of the ferronematic, i.e., a formation of large multiparticle aggregates whose total magnetic moment is close to zero [11]. These aggregates would be insensitive to a weak external magnetic field, thus reducing the birefringence effect.

7. Discussion. In this paper we have carried out an exhaustive analysis of the ordering processes which take place in a Frederiks-like cell when the liquid crystal is doped by magnetic colloidal particles. These systems are otherwise known as ferronematics. The original motivation for introducing the magnetic particles is to amplify the otherwise low magnetic response. The magnetic Frederiks transition would then be observable at experimentally accessible fields, and the effect could be utilized in magnetically switched liquid crystal devices.

Our calculations show that the simple amplification picture outlined in the last paragraph is at best a great simplification. The mathematical structure of these ferronematic systems seems extremely simple to formulate. There is also an interesting homogenization problem concerned with determining the magnitude of the effective magnetic-nematic director interaction which we have not addressed here, but which is under study elsewhere [25]. The simple formulation nevertheless exhibits a complex and rich set of behaviors as a function of magnetic field, colloidal particle structure, and colloidal concentration.

Although these behaviors can be analyzed easily using computational solutions, we have sought here where possible to examine the structural predictions of the model using mathematical tools. Without this perspective, the model predictions may look counterintuitive. A particular advantage of the method presented here, as opposed to earlier attempts at the same problem, is a change of scaling. The result of this apparently trivial scaling change is that temperature effects on the colloidal density can be added as a perturbation rather than present themselves as an essential element in the theory. 
We find that low magnetic fields do indeed produce a switching analogous to the switching in Frederiks cells in an electric field. However, if the colloidal particles are insufficiently anisotropic, or if their volume fraction is too low, the alignment at low fields is followed by a disorientation process at high fields. In this circumstance, even though the magnetic particles are ordered by the field, the nematic order is dominated by the boundaries at high and low fields, although not at intermediate fields. We have further shown that a first-order disorientation transition is expected as a result of coupling between the orientation and segregation of the magnetic particles into regions where the magnetic force is highest.

The key difference between the ferronematic-induced effects and the direct magnetic effects is the fact that at high fields the magnitude of the ferronematic effects saturates. For very high fields, the director is more strongly tied to the magnetic field than it is to the nematic director. Our treatment completely ignores the direct interaction between the nematic and the magnetic fields. This is normally down by several orders of magnitude, but in the very high field limit, this will no longer be the case. In this very high field limit, our high field asymptotics would need to be modified. In extreme cases, where the direct and induced fields compete, there is further potentially interesting very high field physics. But we anticipate that this will occur at experimentally inaccessible magnetic fields. In any event, we postpone this to future work.

Finally, we note again the paucity of experimental data on these systems. This is a result of the difficulties of aligning magnetic particles themselves at higher temperatures, and also of preventing van der Waals forces from forcing irreversible colloidal aggregation. Attempts in the physics and engineering communities to make progress in this area continue. If these attempts bear fruit, there are further interesting mathematical problems to attack in this area. The most obvious of these is the dynamics of the ordering process itself, which, because of the nature of the couple exerted by the magnetic field on the local dipole moment, could in principle lead to a slow and oscillatory response. We postpone this problem to a future paper.

Acknowledgments. We acknowledge useful discussions over the course of this work with M. P. Allen, S. V. Burylov, M. Carme Calderer, D. Golovaty, I. P. Pinkevich, Y. Reznikov, C. Rosenblatt, and A. N. Vasilev.

\section{REFERENCES}

[1] F. Brochard and P. G. De Gennes, Theory of magnetic suspensions in liquid crystals, J. Physique (France), 31 (1970), pp. 691-708.

[2] C. Mauguin, Orientation of liquid crystals by a magnetic field, C. R. Acad. Sci., 152 (1911), pp. 1680-1683 (in French); translation appears in Crystals that Flow: Classic Papers from the History of Liquid Crystals, T. J. Sluckin, D. A. Dunmur, and H. Stegemeyer, eds., Taylor and Francis, London, 2004, pp. 122-127.

[3] S.-H. Chen And N. M. Amer, Observation of macroscopic collective behavior and new texture in magnetically doped liquid crystals, Phys. Rev. Lett., 51 (1983), pp. 2298-2301.

[4] S. K. Srivatsa and G. S. Ranganath, Nematic kink states in a laser field, Phys. Rev. E (3), 60 (1999), pp. 5639-5646.

[5] C. Y. Matuo and A. M. Figueiredo Neto, Time dependence of the magnetic grain concentration and secondary grain aggregation in ferronematic lyotropic liquid crystals subjected to magnetic field gradients, Phys. Rev. E (3), 60 (1999), pp. 1815-1820.

[6] P. Kopčanský, I. Potočová, M. Timko, M. Koneracké, A. M. G. Jansen, J. Jadzyn, AND G. CZECHOWSKI, The structural transitions in ferronematics in combined electric and magnetic fields, J. Magn. Magn. Mater., 272-276 (2004), pp. 2355-2356.

[7] Yu. L. Raikher and V. I. Stepanov, Transient field-induced birefringence in a ferronematic, J. Magn. Magn. Mater., 201 (1999), pp. 182-185. 
[8] V. Berejnov, J.-C. Bacri, V. Cabuil, R. Perzynski, and Yu. Raikher, Lyotropic ferronematics: Magnetic orientational transition in the discotic phase, Europhys. Lett., 41 (1998), pp. 507-512.

[9] O. Buluy, E. Ouskova, Yu. Reznikov, and P. Litvin, Preparation and properties of a ferromagnetic nematic suspension, Ukr. J. Phys., 49 (12A) (2004), pp. A48-A50.

[10] S. V. Burylov and YU. L. Raikher, Macroscopic properties of ferronematics caused by orientational interactions on the particle surfaces I: Extended continuum model, Mol. Cryst. Liq. Cryst., 258 (1995), pp. 107-122.

[11] S. V. BuRYlov and Yu. L. RaikheR, Macroscopic properties of ferronematics caused by orientational interactions on the particle surfaces II: Behavior of real ferronematics in external fields, Mol. Cryst. Liq. Cryst., 258 (1995), pp. 123-141.

[12] B. J. LIANG AND S.-H. Chen, Electric-field-induced molecular reorientation of a magnetically biased ferronematic liquid-crystal film, Phys. Rev. A (3), 39 (1989), pp. 1441-1446.

[13] S. V. Burylov, V. I. Zadorozhnit, I. P. Pinkevich, V. Yu. Reshetnyak, and T. J. Sluckin, Weak anchoring effects in ferronematic systems, J. Magn. Magn. Mater., 252 (2002), pp. $153-155$.

[14] V. I. Zadorozhnit, I. P. Pinkevich, V. Yu. Reshetnyak, S. V. Burylov, and T. J. Sluckin, Adsorption phenomena and macroscopic properties of ferronematics caused by orientational interactions, Mol. Cryst. Liq. Cryst., 409 (2004), pp. 285-292.

[15] V. I. Zadorozhni, A. N. Vasilev, V. Yu. Reshetnyak, K. S. Thomas, and T. J. Sluckin, Nematic director response in ferronematic cells, Europhys. Lett., 73 (2006), pp. 408-414.

[16] B. I. Lev, S. B. Chernyshuk, P. M. Tomchuk, And H. Yokoyama, Symmetry breaking and interaction of colloidal particles in nematic liquid crystals, Phys. Rev. E (3), 65 (2002), 021709 .

[17] L. M. Blinov and V. G. Chigrinov, Electrooptic Effects in Liquid Crystal Materials, SpringerVerlag, New York, 1994.

[18] A. N. Zakhlevnykh, Threshold magnetic fields and Fréedericksz transition in a ferronematic, J. Magn. Magn. Mater., 269 (2004), pp. 238-244.

[19] R. H. Self, C. P. Please, and T. J. Sluckin, Deformation of nematic liquid crystals in an electric field, European J. Appl. Math., 13 (2002), pp. 1-23.

[20] E. G. Virga, Variational Theories for Liquid Crystals, Chapman and Hall, London, 1994.

[21] Z. Wang AND C. Holm, Structure and magnetic properties of polydisperse ferrofluids: A molecular dynamics study, Phys. Rev. E (3), 68 (2003), 041401.

[22] L. L. Afremov and A. V. Panov, Magnetic states and hysteresis properties of small magnetite particles, The Physics of Metals and Metallography, 86 (1998), pp. 269-275.

[23] P. Poulin, V. Cabuil, and D. A. Weitz, Direct measurement of colloidal forces in an anisotropic solvent, Phys. Rev. Lett., 79 (1997), pp. 4862-4865.

[24] B. I. Halperin, T. C. Lubensky, and S. K. Ma, First-order phase transitions in superconductors and smectic-A liquid crystals, Phys. Rev. Lett., 32 (1974), pp. 292-295.

[25] M. Carme Calderer and D. Golovaty, Private communication, University of Minnesota, 2007.

Copyright (c) by SIAM. Unauthorized reproduction of this article is prohibited. 